\title{
Weak chaos, infinite ergodic theory, and anomalous dynamics
}

\author{
Rainer Klages \\ Queen Mary University of London \\ School of Mathematical Sciences \\ Mile End Road, London E1 4NS \\ e-mail: r.klages@qmul.ac.uk
}

\begin{abstract}
This book chapter introduces to the concept of weak chaos, aspects of its ergodic theory description, and properties of the anomalous dynamics associated with it. In the first half of the chapter we study simple one-dimensional deterministic maps, in the second half basic stochastic models and eventually an experiment. We start by reminding the reader of fundamental chaos quantities and their relation to each other, exemplified by the paradigmatic Bernoulli shift. Using the intermittent Pomeau-Manneville map the problem of weak chaos and infinite ergodic theory is outlined, defining a very recent mathematical field of research. Considering a spatially extended version of the Pomeau-Manneville map leads us to the phenomenon of anomalous diffusion. This problem will be discussed by applying stochastic continuous time random walk theory and by deriving a fractional diffusion equation. Another important topic within modern nonequilibrium statistical physics are fluctuation relations, which we investigate for anomalous dynamics. The chapter concludes by showing the importance of anomalous dynamics for understanding experimental results on biological cell migration.
\end{abstract}




\section{Contents}

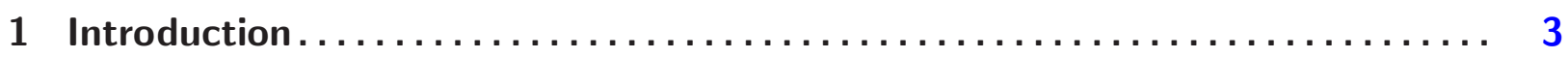

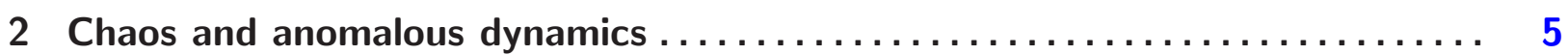

2.1 Deterministic chaos in a simple map . . . . . . . . . . . . . . . 5

2.2 Weak chaos and infinite ergodic theory . . . . . . . . . . . . . . 7

2.3 A generalized hierarchy of chaos . . . . . . . . . . . . . . . . 12

3 Anomalous diffusion $\ldots \ldots \ldots \ldots \ldots \ldots \ldots \ldots \ldots \ldots \ldots \ldots \ldots \ldots \ldots \ldots \ldots \ldots \ldots \ldots, 13$

3.1 A simple model generating anomalous diffusion . . . . . . . . . . . . . . 14

3.2 Continuous time random walk theory . . . . . . . . . . . . . . 15

3.3 A fractional diffusion equation . . . . . . . . . . . . . . . . . . . . . . 19

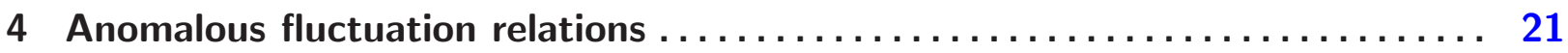

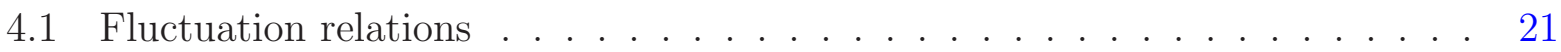

4.2 Fluctuation relations for ordinary Langevin dynamics . . . . . . . . . . . . . . 22

4.3 Fluctuation relations for anomalous Langevin dynamics . . . . . . . . . . . . 24

5 Anomalous dynamics of biological cell migration $\ldots \ldots \ldots \ldots \ldots \ldots \ldots \ldots 27$

5.1 Cell migration . . . . . . . . . . . . . . . . . . . . . . 27

5.2 Experimental results and statistical analysis . . . . . . . . . . . . . 27

5.3 Stochastic modeling . . . . . . . . . . . . . . . . . 29

6 Summary $\ldots \ldots \ldots \ldots \ldots \ldots \ldots \ldots \ldots \ldots \ldots \ldots \ldots \ldots \ldots \ldots \ldots \ldots \ldots \ldots \ldots \ldots \ldots \ldots, 32$

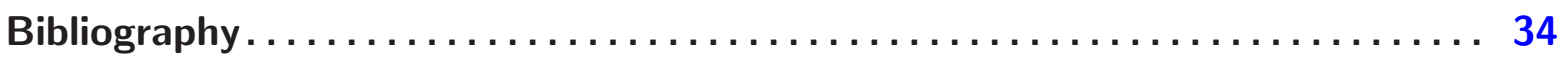




\section{Introduction}

Deterministic dynamical systems involving only a few variables can exhibit complexity reminiscent of many-particle systems if the dynamics is chaotic, as is quantified by the existence of a positive Lyapunov exponent [Sch89b, Ott93, Bec93, Al197]. Such systems, which may be called small because of their small number of degrees of freedom [Kla12b], can display an intricate interplay between nonlinear microscopic dynamical properties and macroscopic statistical behavior leading to highly non-trivial fluctuations of physical observables. This becomes particularly interesting in nonequilibrium situations when these systems are exposed to external gradients or fields. Despite their complexity, examples of these systems are still amenable to detailed analysis by means of dynamical systems theory in combination with stochastic theory. Hence, they provide important paradigms to construct a theory of nonequilibrium statistical physics from first principles: Based on the chaotic hypothesis, which generalizes Boltzmann's ergodic hypothesis, SRB measures were studied as nonequilibrium equivalents of the Gibbs ensembles of equilibrium statistical mechanics. This novel approach led to the discovery of fundamental relations characterizing nonequilibrium transport in terms of microscopic chaos [Dor99, Gas98, Kla07, Cas08], such as formulas expressing transport coefficients in terms of Lyapunov exponents and dynamical entropies, equations relating the nonequilibrium entropy production to the fractality of SRB measures, and fluctuation relations, which are now widely studied as a fundamental property of nonequilibrium processes [Eva02, Bus05, Kla07, Kla12b].

The interplay between these different levels of description in modern nonequilibrium statistical mechanics is illustrated by the second column in Fig. 1.1, in analogy to the theory of equilibrium statistical mechanics sketched in the first column. As is represented by the third column, however, more recently scientists learned that random-looking evolution in time and space also occurs under conditions that are weaker than requiring a positive Lyapunov exponent [Aar97, Zas01]. It is now known that there is a wealth of systems exhibiting zero Lyapunov exponents, meaning that the separation of nearby trajectories is weaker than exponential. This class of dynamical systems is called weakly chaotic. Examples include maps with indifferent fixed points, polygonal particle billiards, and Hamiltonian systems with sticky islands in phase space [Aar97, Zas01, Kla07, Kla08].

Weakly chaotic systems exhibit anomalous dynamics characterized by novel properties such as ageing, which reflects an extremely weak relaxation towards equilibrium involving more than one time scale in the decay of correlations. Other surprising properties are the existence of Lévy-type probability distributions obeying generalized central limit theorems [Shl93, Kla96a] and the non-equivalence of time and ensemble averages, called weak ergodicity breaking [Ste09]. These physical phenomena were observed experimentally in a wide variety of systems, such as in the anomalous statistics of blinking quantum dots, in the anomalous diffusion of atoms in optical lattices, in plasma physics and even in cell and animal migration [Met00, Met04, Kla08, Ste09]. 


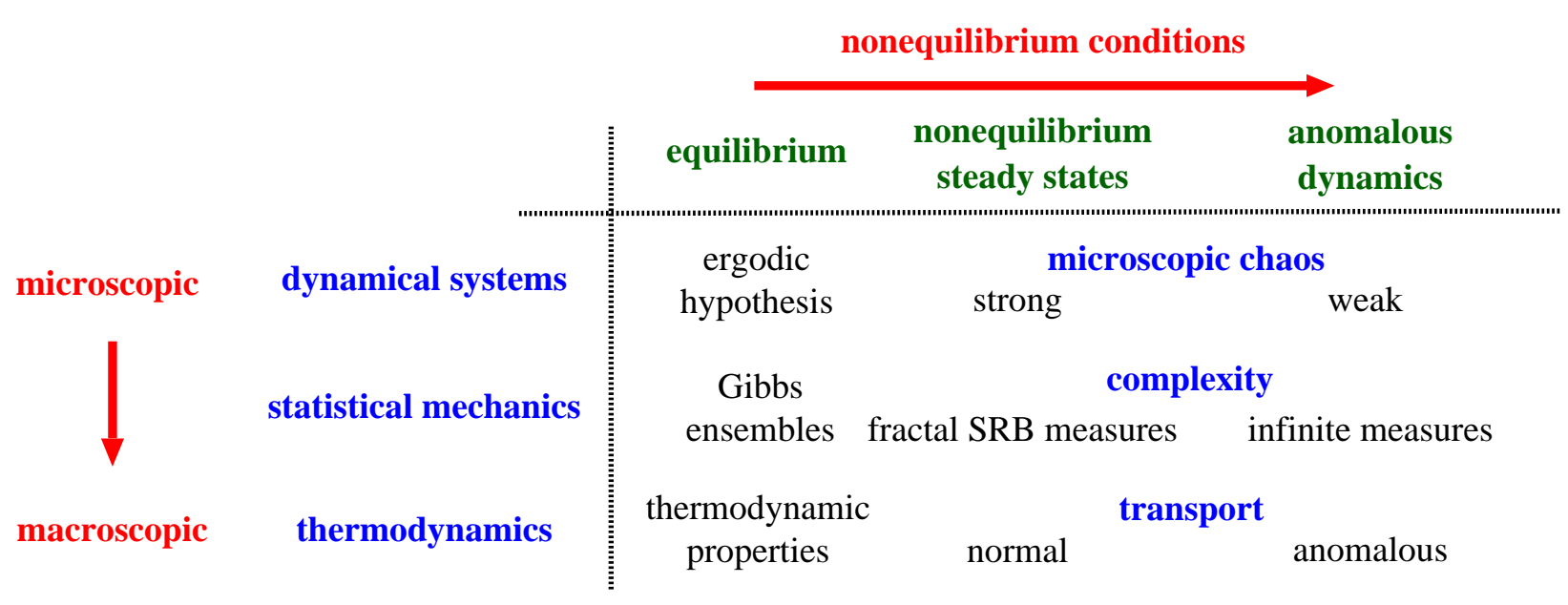

Figure 1.1: Conceptual foundations of a theory of nonequilibrium statistical physics based on dynamical systems theory by motivating the topic of this book chapter, which is represented by the third column.

Recent work in ergodic theory, on the other hand, has led to mathematically rigorous results about some of the physically relevant phenomena mentioned above. It turned out that there is an intimate connection between the mechanism generating weakly chaotic dynamics and the existence of non-normalizable, so-called infinite invariant measures [Aar97, Aar00, Tha06]. The ergodic theory of generalized random walks driven by weak chaos and of other systems exhibiting infinite invariant measures, which is called infinite ergodic theory, has thus the potential of providing a sound mathematical basis for some of the physical phenomena displayed by anomalous dynamics.

This book chapter gives a brief introduction to important aspects of the above topics in four sections: As a warm-up, the beginning of Section 2 briefly reminds us of the concept of deterministic chaos in simple dynamical systems as quantified by a positive Lyapunov exponent. On this basis, we will introduce to the phenomenon of weak chaos, and the idea of infinite ergodic theory will be outlined. The chapter concludes by putting different forms of deterministic chaos into perspective. Section 3 relates these concepts and ideas to the problem of anomalous diffusion in deterministic systems. Here we make a transition to stochastic theory by studying these systems also from a stochastic point of view. For this purpose we use a generalization of ordinary random walk theory, called continuous time random walk theory. In a scaling limit, this theory leads to generalized diffusion equations involving fractional derivatives. Section 4 introduces to the topic of fluctuation relations, which generalize the Second Law of Thermodynamics and other fundamental thermodynamic relations to small systems far away from equilibrium. After discussing transient fluctuation relations for a very basic type of stochastic dynamics as an example, we explore the validity of such relations for generalizations of this dynamics yielding anomalous diffusion. In section 5 we relate this line of theoretical reasoning about anomalous dynamics to biophysical reality by studying the case of biological cell migration. After briefly introducing to the problem of cell migration, we report experimental results on fundamental statistical physical properties of migrating cells, extracted from statistical data analysis. We conclude this section with a stochastic modeling of these experimental results by using a generalized, fractional Fokker-Planck type equation. We summarize our discussion of this book chapter in the final section 6 . 
The title of this review is inspired by a conference that the author had the pleasure to organize together with R.Zweimüller, E.Barkai, and H.Kantz at the Max Planck Institute for the Physics of Complex Systems, Dresden, in Summer 2011, which bears exactly the same title [Kla11]. However, naturally this chapter reflects the author's very personal take on this topic, and his own research. The subsequent second section is to some extent based on the review Ref. [Kla10] by combining it with ideas from Refs. [Kla07, How09]. The third section builds on Refs. [Kor05, Kor07]. The fourth section incorporates material from the review Ref. [Kla12a] and from Ref. [Che09], the fifth one draws on Ref. [Die08].

\section{Chaos and anomalous dynamics}

In this section we focus on purely deterministic dynamics modeled by two simple but paradigmatic one-dimensional maps: the famous Bernoulli shift, as a model for strong chaos characterized by a positive Lyapunov exponent, and the Pomeau-Manneville map, as an example exhibiting weak chaos with zero Lyapunov exponent. We start by briefly reminding of basic concepts of dynamical systems theory and ergodic theory such as Lyapunov exponents, ergodicity, SRB measures, and Pesin's theorem, illustrated for the Bernoulli shift. Ref. [Kla10] provides a more tutorial exposition of most of these ideas. By switching to the Pomeau-Manneville map we find that generalizations of these concepts are needed in order to describe the model's weakly chaotic dynamics. This motivates the mathematical problem of infinite ergodic theory, which is intimately related to defining suitably generalized chaos quantities assessing weak chaos, and a generalization of Pesin's theorem. In the final part of this chapter we propose a generalized hierarchy of chaos, based on the existence of different types of stretching between two nearby trajectories, which we use to characterize chaotic dynamics.

\subsection{Deterministic chaos in a simple map}

The main vehicle of our approach in this and the next section are one-dimensional timediscrete maps $F: J \rightarrow J, J \subseteq \mathbb{R}$ obeying

$$
x_{n+1}=F\left(x_{n}\right), n \in \mathbb{N}_{0},
$$

which defines the equations of motion of our deterministic dynamical systems. For a given initial condition $x_{0}$ we have $x_{n}=F^{n}\left(x_{0}\right)$. A particularly simple example of $F$ are piecewise linear maps, such as the paradigmatic Bernoulli shift [Sch89b, Ott93, Al197, Dor99]

$$
B:[0,1) \rightarrow[0,1), B(x):=2 x \bmod 1=\left\{\begin{array}{l}
2 x, 0 \leq x<1 / 2 \\
2 x-1,1 / 2 \leq x<1
\end{array}\right.
$$

depicted in Fig. 2.1. This simple system exhibits a very complicated dynamics governed by sensitivity to initial conditions, as can be quantified by calculating its Lyapunov exponent 


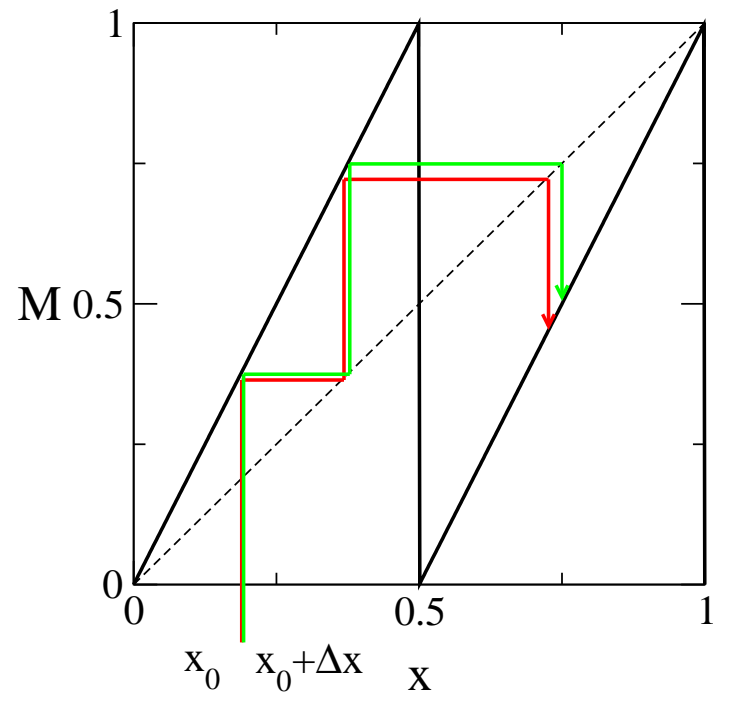

Figure 2.1: The Bernoulli shift Eq. (2.2) and two trajectories starting from two nearby initial conditions $x_{0}$ and $x_{0}^{\prime}=x_{0}+\Delta x_{0}$ displaced by $\Delta x_{0} \ll 1$.

[Ott93, Rob95]: Consider two points that are initially displaced from each other by $\Delta x_{0}:=$ $\left|x_{0}^{\prime}-x_{0}\right|$ with $\Delta x_{0}$ "infinitesimally small" such that $x_{0}, x_{0}^{\prime}$ do not hit different branches of the Bernoulli shift $B(x)$ around $x=1 / 2 .{ }^{1}$ We then have

$$
\Delta x_{n}:=\left|x_{n}^{\prime}-x_{n}\right|=2 \Delta x_{n-1}=2^{2} \Delta x_{n-2}=\ldots=2^{n} \Delta x_{0}=e^{n \ln 2} \Delta x_{0} \quad .
$$

We thus see that there is an exponential separation between two nearby points as we follow their trajectories, where the rate of separation $\lambda\left(x_{0}\right):=\ln 2$ is the (local) Lyapunov exponent of $B(x)$. Since $\lambda\left(x_{0}\right)>0$, the system displays an exponential dynamical instability and is hence called chaotic (in the sense of Lyapunov) [Rob95, Ott93, All97, Bec93].

Writing down the analogue of Eq. (2.3) for a given differentiable map $F$, we get

$$
\Delta x_{n}=\left|x_{n}^{\prime}-x_{n}\right|=\left|F^{n}\left(x_{0}^{\prime}\right)-F^{n}\left(x_{0}\right)\right|=: e^{n \lambda\left(x_{0}\right)} \Delta x_{0}\left(\Delta x_{0} \rightarrow 0\right),
$$

which we can take as the definition of the Lyapunov exponent $\lambda\left(x_{0}\right)$ that comes in as the exponential stretching rate on the right hand side. Solving this equation for $\lambda\left(x_{0}\right)$ by using the chain rule, it is not too hard to see [Al197] that this simple procedure of calculating $\lambda$ can be generalized in terms of the time (or Birkhoff) average

$$
\lambda(x)=\lim _{n \rightarrow \infty} \frac{1}{n} \sum_{i=0}^{n-1} \ln \left|F^{\prime}\left(x_{i}\right)\right|
$$

with $x=x_{0}$. If the dynamical system defined by the map $F$ is ergodic, the time average does not depend on the initial condition for a typical $x, \lambda=\lambda(x)=$ const. It can be shown that the Bernoulli shift is ergodic [Dor99], and indeed, following Eq. (2.5), for $B$ we trivially find that $\lambda=\ln 2$ for all $x$. In particular, according to Birkhoff's theorem [Dor99, Kat95, Arn68, Tod92], for ergodic systems the time average is equal to the ensemble average, which for the Lyapunov exponent of one-dimensional maps reads

$$
\lambda=\left\langle\ln \left|F^{\prime}(x)\right|\right\rangle_{\mu^{*}}:=\int_{J} d \mu^{*} \ln \left|F^{\prime}(x)\right| .
$$

\footnotetext{
${ }^{1}$ This condition could be eliminated by defining a metric on a circle [All97].
} 
Here $\mu^{*}$ is the invariant measure of the map. If the map exhibits an SRB measure [You02, Eck85, Bal00], we have

$$
d \mu^{*}=\rho^{*}(x) d x,
$$

where $\rho^{*}(x)$ holds for the invariant density of the map. That is, the measure $\mu^{*}$ has the nice property that it can be obtained by integrating a density,

$$
\mu^{*}(A)=\int_{A} d x \rho^{*}(x), A \subseteq J
$$

which simplifies the calculation of the ensemble average Eq. (2.6). For the Bernoulli shift it is not too difficult to see [Bec93] that, for typical initial conditions, the invariant density is $\rho^{*}(x)=1$. By combining Eqs. (2.6) and (2.7), we get

$$
\lambda=\int_{0}^{1} d x \rho^{*}(x) \ln 2=\ln 2 .
$$

This result is equal to the time average calculated above and confirms the result obtained from our handwaving argument Eq. (2.3).

Lyapunov exponents are not the only quantities assessing the chaotic character of a dynamical system. Pesin's Theorem [Dor99, Eck85, You02] states that for closed $C^{2}$ Anosov [Eck85, Dor99] systems the Kolmogorov-Sinai (or metric) entropy $h_{K S}$ is equal to the sum of positive Lyapunov exponents. For one-dimensional maps that are expanding [A1197, Bec93],

$$
\forall x \in J \quad\left|F^{\prime}(x)\right|>1
$$

this theorem boils down to

$$
\lambda=h_{K S}
$$

where [Ott93, Dor99]

$$
h_{K S}:=\lim _{n \rightarrow \infty}-\frac{1}{n} \sum_{w \in\left\{W_{i}^{n}\right\}} \mu^{*}(w) \ln \mu^{*}(w) .
$$

Here $\mu^{*}(w)$ is the SRB measure of an element $w$ of the partition $\left\{W_{i}^{n}\right\}$, and $n$ defines the level of refinement of the partition. Note that in Eq. (2.12) we have assumed that the partition is generating [Eck85, Kat95, Bad97]. If $h_{K S}>0$ one sometimes speaks of measuretheoretic chaos [Bec93]. For the Bernoulli shift it is not too hard to calculate $h_{K S}$ from first principles leading to $h_{K S}=\ln 2$ [Ott93, Bec93], which combined with our previous result for the Lyapunov exponent is in line with Pesin's theorem. This theorem can be formulated under weaker assumptions, and it is believed to hold for a wider class of dynamical systems than stated above. We remark that typically the KS-entropy is much harder to calculate for a given dynamical system than Lyapunov exponents. Hence, Pesin's theorem is often employed in the literature for indirectly calculating the KS-entropy.

\subsection{Weak chaos and infinite ergodic theory}

Let us now consider a nonlinear generalization of our previous piecewise linear model, which is known as the Pomeau-Manneville map [Pom80],

$$
P_{a, z}(x)=x+a x^{z} \bmod 1,
$$




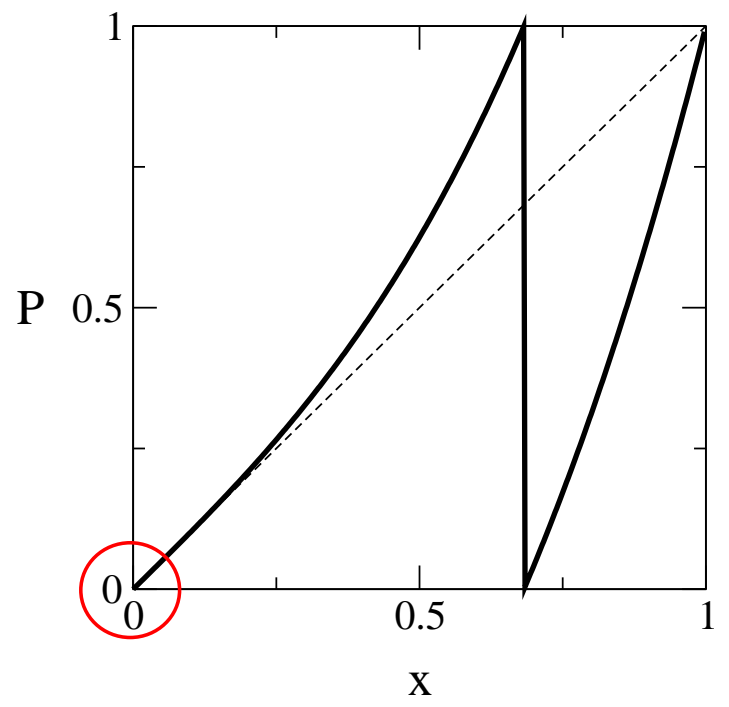

Figure 2.2: The Pomeau-Manneville map Eq. (2.13) for $a=1$ and $z=3$. Note that there is a marginal fixed point at $x=0$ leading to the intermittent behavior depicted in Fig. 2.3.

see Fig. 2.2, where following Eq. (2.1) the dynamics is defined by $x_{n+1}=P_{a, z}\left(x_{n}\right)$. This map has the two control parameters $a \geq 1$ and the exponent of nonlinearity $z \geq 1$. For $a=1$ and $z=1$ the map reduces to the Bernoulli shift Eq. (2.2), for $z>1$ it provides a nontrivial nonlinear generalization of it. The nontriviality is due to the fact that in this case the stability of the fixed point at $x=0$ becomes marginal (sometimes also called indifferent, or neutral), $P_{a, z}^{\prime}(0)=1$. This implies that the map is non-hyperbolic, because [Dev89],

$$
\nexists N>0 \text { such that } \forall x \forall n \geq N\left|\left(P_{a, z}^{n}\right)^{\prime}(x)\right| \neq 1,
$$

which is related to the fact that the map is not expanding anymore according to Eq. (2.10). Since the map is smooth around $x=0$, the dynamics resulting from the left branch of the map is determined by the stability of this fixed point, whereas the right branch is still of 'Bernoulli shift-type' generating ordinary chaotic dynamics. There is thus a competition in the dynamics between these two different branches as illustrated by the time series displayed in Fig. 2.3: One can observe that long periodic 'laminar phases' determined by the marginal fixed point around $x=0$ are interrupted by 'chaotic bursts' reflecting the Bernoulli shifttype part of the map with slope $a>1$ around $x=1$. This phenomenology is the hallmark of what is called intermittency [Sch89b, Ott93].

This seemingly small nonlinear modification of the Bernoulli shift has dramatic consequences for the whole dynamics of the new system. We discuss them step by step following our exposition of the Bernoulli shift dynamics in Section 2.1: The invariant density of the Pomeau-Manneville map can be calculated to [Tha83, Zwe98, Kor09, Kor10, How09]

$$
\rho^{*}(x) \sim x^{1-z}(x \rightarrow 0) .
$$

This singularity reflects the stickiness of trajectories to the marginally unstable fixed point at $x=0$. Correspondingly, the measure obtained by integrating this density is nonnormalizable for $z \geq 2$ yielding the infinite invariant measure

$$
\mu^{*}(x)=\int_{x}^{1} d y \rho^{*}(y) \rightarrow \infty(x \rightarrow 0) .
$$




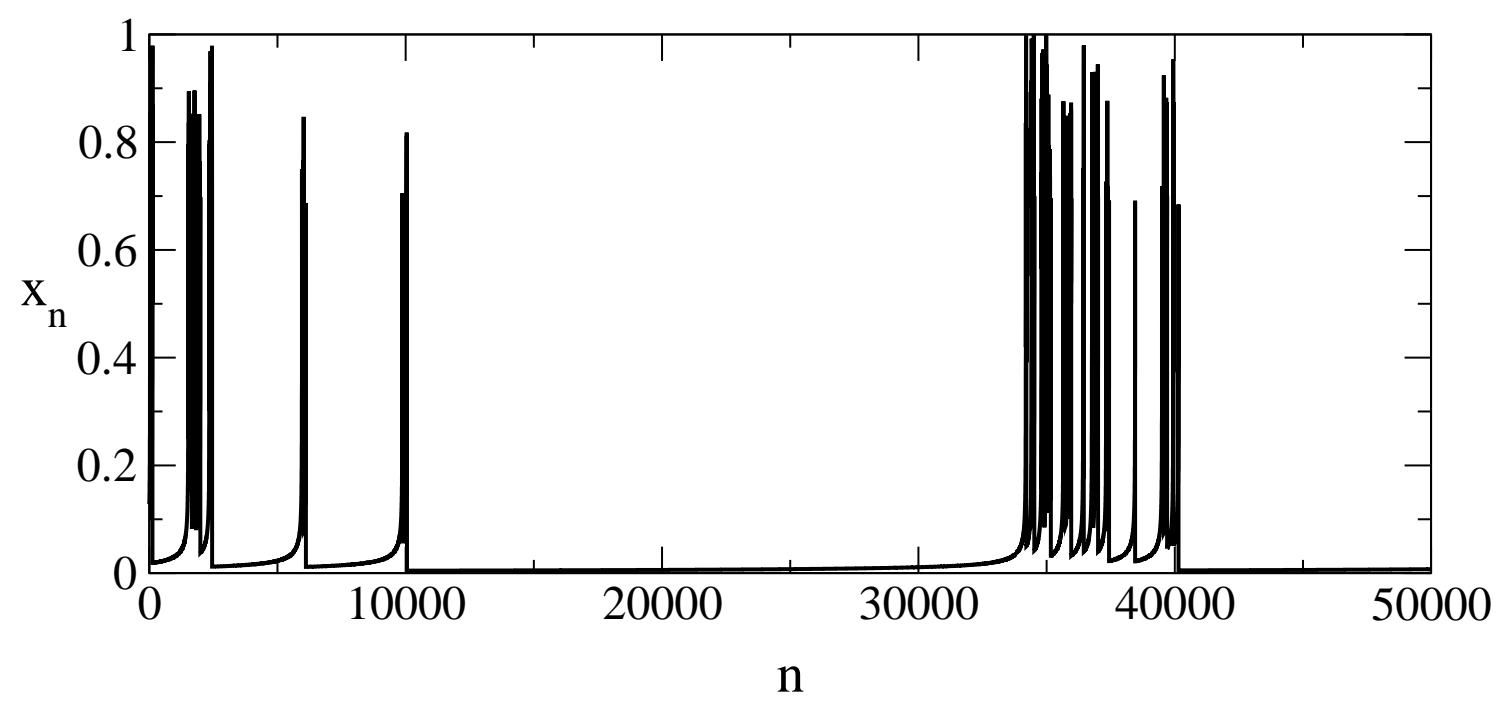

Figure 2.3: Phenomenology of intermittency in the Pomeau-Manneville map Fig. 2.2: The plot shows the time series of position $x_{n}$ versus discrete time step $n$ for an orbit generated by the map Eq. (2.13), which starts at a typical initial condition $x_{0}$.

The branch of erogdic theory exploring the ergodic properties of infinite measure preserving dynamical systems is thus called infinite ergodic theory; see Refs. [Tha01, Aar00, Zwe09] for introductions to this topic and [Aar97] for an in-depth mathematical treatment. The marginal fixed point has also an impact on the dispersion of nearby trajectories, which can be calculated to [Gas88, Kor09, Kor10, How09]

$$
\Delta x_{n} \sim \exp \left(n^{\frac{1}{z-1}}\right) \Delta x_{0}(z>2) .
$$

In contrast to the Bernoulli shift, which according to Eq. (2.3) exhibits exponential sensitivity to intial conditions, here we thus have a weaker stretched exponential sensitivity. By repeating the calculation leading to Eq. (2.5), it is not hard to see that Eq. (2.17) yields a zero Lyapunov exponent,

$$
\lambda=0,
$$

despite the fact that Fig. 2.3 displays irregular dynamics. Dynamical systems where the separation of nearby trajectories grows weaker than exponential, which implies that the corresponding Lyapunov exponents are zero, have been coined weakly chaotic [Zas01, Gal03, vB04, Art05]. We remark, however, that this denotation is not used unambiguously in the literature. Most importantly, the standard definitions of Lyapunov exponents for expanding and hyperbolic systems Eqs. (2.5),(2.6) yield no good indicators of irregular dynamics anymore, because they do not capture the sub-exponential dispersion of trajectories. It is thus desirable to come up with generalized definitions of ordinary chaos quantities, which enable us to still assess this different type of chaotic behavior by calculating quantities that yield non-zero values.

The way to achieve this goal is shown by advanced concepts of infinite ergodic theory and corresponding generalized ergodic theorems. Recall that Birkhoff's theorem implies that for observables which are Lebesgue integrable, $f \in L^{1}$, we have [Dor99, Kat95, Arn68, Tod92]

$$
\frac{1}{n} \sum_{i=0}^{n-1} f\left(x_{i}\right)=\langle f\rangle_{\mu^{*}} .
$$


However, it turns out that for $z \geq 2$ the Birkhoff sum on the left hand side does not converge anymore. Surprisingly, it becomes a random variable that depends on initial conditions, and the equation breaks down. This non-equivalence between time and ensemble averages became known as weak ergodicity breaking in the physics literature, see, e.g., Refs. [Reb08, Kla08, Ste09] and further references therein. It was observed experimentally in the anomalous statistics of blinking quantum dots and plays also a crucial role for the anomalous diffusion of atoms in optical lattices [Kla08, Reb08, Ste09]. Note that physicists typically refer to ergodicity as the equality between time and ensemble average, whereas mathematicians usually define ergodicity via indecomposability [Arn68, Kat95]. Eq. (2.19) then follows from this definition by using Birkhoff's theorem. This should be kept in mind when referring to a weak ergodicity breaking.

In case of $z \geq 2$ and $f \in L^{1}$ for our map, the nature of the breakdown of Eq. (2.19) is elucidated by the Aaronson-Darling-Kac theorem [Zwe00, Tha06, Zwe09]

$$
\frac{1}{a_{n}} \sum_{i=0}^{n-1} f\left(x_{i}\right) \stackrel{d}{\rightarrow} \mathcal{M}_{\alpha}\langle f\rangle_{\mu^{*}}(n \rightarrow \infty),
$$

where the arrow holds for convergence in distribution. Here $\mathcal{M}_{\alpha}, \alpha \in[0,1]$, denotes a non-negative real random variable distributed according to the normalized Mittag-Leffler distribution of order $\alpha$, which is characterized by its moments

$$
\left\langle\mathcal{M}_{\alpha}^{r}\right\rangle=r ! \frac{(\Gamma(1+\alpha))^{r}}{\Gamma(1+r \alpha)}, r \geq 0 .
$$

For the Pomeau-Manneville map $P_{a, z}$ one can prove [Zwe00] that $a_{n} \sim n^{\alpha}$ with $\alpha:=1 /(z-1)$. Integrating Eq. (2.21) with respect to Lebesgue measure $m$ suggests

$$
\frac{1}{n^{\alpha}} \sum_{i=0}^{n-1}\left\langle f\left(x_{i}\right)\right\rangle_{m} \sim\langle f\rangle_{\mu^{*}} .
$$

Note that for $z<2$ one has to choose $\alpha=1$, because the map still exhibits an SRB measure, and Eq. (2.22) becomes an equality. However, for $z \geq 2$ we have an infinite invariant measure that cannot be normalized, hence here Eq. (2.22) remains a proportionality, unless we fix this constant by other constraints.

These known facts from infinite ergodic theory motivate to suitably define generalized chaos quantities, which assess weakly chaotic dynamics by yielding non-zero values. Following the left hand side of Eq. (2.22), by choosing $f(x)=\ln \left|P_{a, z}^{\prime}(x)\right|$ we define the generalized Lyapunov exponent as

$$
\Lambda:=\lim _{n \rightarrow \infty} \frac{\Gamma(1+\alpha)}{n^{\alpha}} \sum_{i=0}^{n-1}\left\langle\ln \left|M^{\prime}\left(x_{i}\right)\right|\right\rangle_{m} .
$$

The inclusion of the gamma function in the numerator is not obvious at this point, however, it turns out to be convenient when calculating $\Lambda$ for the Pomeau-Manneville map [How09]. Interestingly, it is precisely the same canonical choice as is made in other areas of anomalous dynamics [Kor07]. Analogously, we amend Eq. (2.12) to define the generalized KS entropy as

$$
H_{K S}:=\lim _{n \rightarrow \infty}-\frac{\Gamma(1+\alpha)}{n^{\alpha}} \sum_{w \in\left\{W_{i}^{n}\right\}} \mu^{*}(w) \ln \mu^{*}(w) .
$$




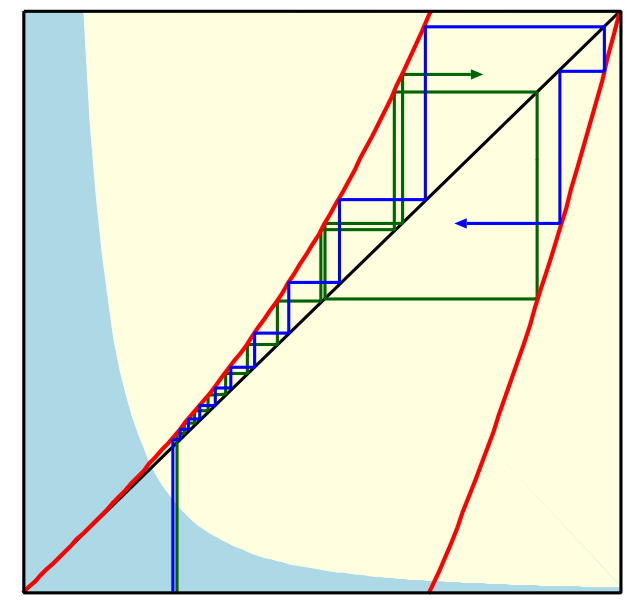

Figure 2.4: Illustration of the interplay between weak chaos, infinite measures, and anomalous dynamics in the Pomeau-Manneville map Eq. (2.13) shown by the thick red lines: Anomalous dynamics is indicated by the irregular behaviour of the two single trajectories. Weak chaos is exemplified by the divergence of the two trajectories starting at nearby initial conditions. The light blue area in the lower left corner depicts the shape of the infinite invariant density, which diverges at the marginal fixed point of the map [Kla11].

Both quantities can be calculated independently for the piecewise linearization of $P_{a, z}$ proposed in Ref. [Gas88] by applying the thermodynamic formalism [Rue78, Bec93] in combination with transfer operator methods [Pre92, Tas02, Tas04]. As a result, one obtains [How09]

$$
H_{K S}=\Lambda,
$$

which may be considered as a generalization of Pesin's formula Eq. (2.11) to anomalous dynamics. Related generalizations of chaos quantities, and other versions of a generalized Pesin formula, have been discussed in Refs. [Kor09, Kor10, Aki10, Saa12]. We remark, however, that in the mathematical literature there is the well-known formula by Rokhlin [Kel98], which for the Pomeau-Manneville map reads [Tha83, Zwe00]

$$
h_{K r}=\left\langle\ln \left|P_{a, z}^{\prime}(x)\right|\right\rangle_{\mu^{*}} .
$$

Here the left hand side holds for the so-called Krengel entropy. In case of finite invariant measures one can show that $h_{K S}=h_{K r}$, the right hand is the Lyapunov exponent defined via the ensemble average Eq. (2.6), and Rokhlin's formula boils down to Pesin's formula Eq. (2.11). For infinite invariant measures, one can show that $h_{K r}=H_{K S}$ [How09]. Combining Rokhlin's formula with the integrated form of the Aaronson-Darling-Kac theorem Eq. (2.22) by using $f(x)=\ln \left|P_{a, z}^{\prime}(x)\right|$, exploiting the definition Eq. (2.23) for the generalized Lyapunov exponent, and by fixing the constant of proportionality in Eq. (2.22) with respect to Lebesgue initial measure, one recovers Eq. (2.25). One may thus argue that, within this setting, Rokhlin's formula is a generalization of Pesin's formula for infinite measure-preserving transformations, and that Eq. (2.25) is an illustration of it, worked out for the example of the Pomeau-Manneville map [How09].

The main theoretical objects of discussion in this subsection are shown together in Fig. 2.4. This figure actually represents the logo of the conference about Weak chaos, infinite ergodic theory and anomalous dynamics that was referred to in the introduction [Kla11], from which the title of this book chapter derives. 


\subsection{A generalized hierarchy of chaos}

We conclude this section by embedding the previous results into the more general context of irregular deterministic dynamics [Kla07]. There exist in fact further fundamental types of dynamics that are intermediate between strongly chaotic, in the sense of exponential sensitivity quantified by a positive Lyapunov exponent, and trivially being non-chaotic in terms of purely regular dynamics. These different types of irregular dynamics can be characterized by suggesting a classification of chaotic behavior based on the dispersion of initially infinitesimally close trajectories.

We start from the general expression for the asymptotic growth of the displacement $\Delta(t)$ of two trajectories generated by dynamics in continuous time $t$ in the form of [Gas88]

$$
\ln \Delta(t) \sim t^{\nu_{0}}(\ln t)^{\nu_{1}}, 0 \leq \nu_{0}, \nu_{1} \in \mathbb{R}
$$

If $\nu_{0}=1, \nu_{1}=0$ we recover the usual exponential dynamical instability of Eq. (2.3),

$$
\Delta(t) \sim \exp (\lambda t)
$$

representing Lyapunov chaos [Ott93], whose strength is well quantified by the maximal positive Lyapunov exponent $\lambda$. As discussed before, if $\Delta(t)$ grows weaker than exponential, one speaks of weak chaos [Zas01, Gal03, vB04, Art05]. The regime of Eq. (2.27) with $0<$ $\nu_{0}<1$ or $\nu_{0}=1$ and $\nu_{1}<0$, which is typical for intermittent dynamics, was characterized as sporadic by Gaspard and Wang [Gas88]; cf. Eq. (2.17) and our respective discussion, as well as further details of this dynamics as presented in the following section. Here the dynamical instability is either of stretched exponential type or it is exponential with logarithmic corrections,

$$
\Delta(t) \sim \exp \left(t^{\nu_{0}}(\ln t)^{\nu_{1}}\right) .
$$

Eq. (2.27) with $\nu_{0}=0$ and $\nu_{1}=1$, on the other hand, yields purely algebraic dispersion,

$$
\Delta(t) \sim t^{\nu_{2}}, 0<\nu_{2}
$$

for which Zaslavsky and Edelman [Zas02, Zas03] suggested the term pseudochaos. ${ }^{2}$ Note that algebraic dispersion with logarithmic corrections may also exist,

$$
\Delta(t) \sim t^{\nu_{2}}(\ln t)^{\nu_{3}}, \nu_{3} \in \mathbb{R}
$$

covering a slightly larger class of dynamical systems. A prominent class of dynamical systems exhibiting algebraic dispersion are polygonal billiards; two examples are depicted in Fig. 2.5. They represent the special case of pseudochaotic dynamics with $\nu_{2}=1$ for which the dispersion is strictly linear in time,

$$
\Delta(t) \sim t
$$

However, in contrast to Lyapunov chaos and our weakly chaotic generalizations, here the linear dispersion does not actually capture the essential mechanism leading to dynamical randomness. For example, according to this classification both free flights and polygonal

\footnotetext{
${ }^{2}$ We remark that in Refs. [Zas02, Zas03, Zas05] one finds several slightly different definitions of pseudochaos. Here we refer to the first one stated in Ref. [Zas02].
} 


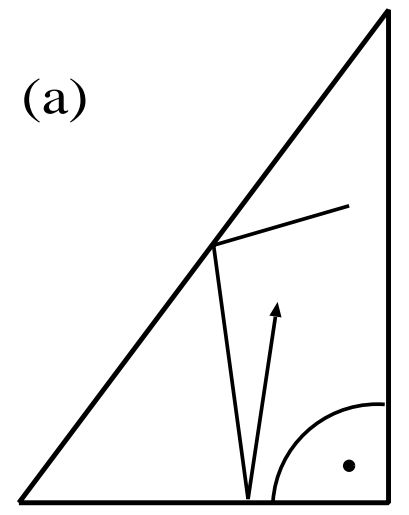

(b)

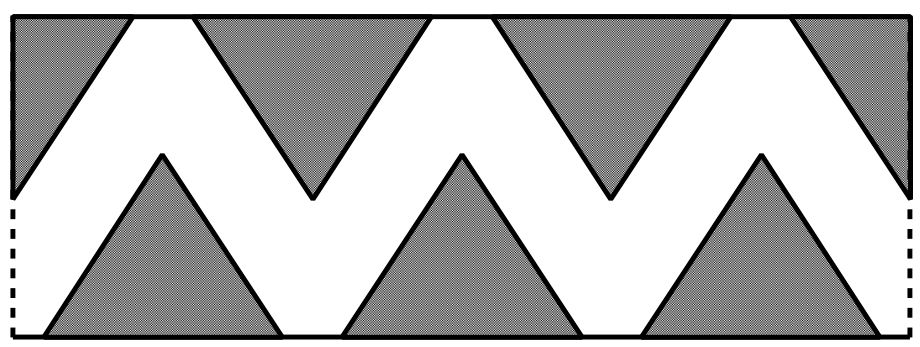

Figure 2.5: Two simple examples of two-dimensional polygonal billiards [Kla07]: A particle with unit velocity moves inside the depicted geometric domains by scattering elastically with their boundaries. (a) shows a right triangular billiard [Art97], (b) the triangle channel, where a unit cell with triangular scatterers is spatially continued along the line [Li02].

billiards of genus one, which clearly exhibit regular dynamics, are also pseudochaotic. As is discussed for the example of rational billiards, e.g., in Ref. [Kla07], in polygonal billiards complicated topologies reflecting the existence of corners, which yield pseudohyperbolic fixed points and pseudointegrability, provide the source of non-trivial irregular dynamics. One is thus tempted to speak of topology-induced chaos ${ }^{3}$ as a subclass of pseudochaos if there is linear dispersion on surfaces that are not integrable. Pseudointegrable rational billiards then form a subclass of topology-induced chaotic dynamical systems. Surprisingly, systems with linear dispersion generating non-trivial dynamics due to complicated topological structures may still exhibit ergodic and transport properties as they are usually associated with Lyapunov unstable chaotic dynamical systems. The trivial end point of this attempt of a generalized classification of chaotic dynamics on the basis of dispersion is simply the purely regular, or periodic, case of $\Delta(t)=$ const.

\section{Anomalous diffusion}

We now establish a cross-link between weakly chaotic dynamics as discussed in the previous section and the problem of deterministic diffusion. The main question we address is what type of diffusion arises if we suitably spatially extend a simple dynamical system exhibiting anomalous dynamics. We first set up our model, which can be considered as a purely deterministic, anomalous version of a random walk on the line, and introduce the concept of anomalous diffusion. We then outline continuous time random walk theory, a standard tool in the theory of stochastic processes to study anomalous diffusion. The results obtained from this theory, worked out for our model, are compared to results from computer simulations. We conclude this chapter by deriving on the basis of this theory a generalized, fractional

\footnotetext{
${ }^{3}$ This should not be confused with topological chaos as defined in Ref. [Gas98].
} 
diffusion equation that approximately reproduces the probability density function (PDF) of our model.

\subsection{A simple model generating anomalous diffusion}

A straightforward way to define a spatially extended dynamical system based on the PomeauManneville map discussed in Section 2.2 is as follows: By using

$$
P_{a, z}(x)=x+a x^{z}, 0 \leq x<\frac{1}{2}
$$

of Eq. (2.13) without the modulus, as well as reflection symmetry,

$$
P_{a, z}(-x)=-P_{a, z}(x),
$$

we continue this map onto the whole real line by a lift of degree one [Fuj82, Gei82, Sch82]

$$
P_{a, z}(x+1)=P_{a, z}(x)+1 .
$$

The resulting model [Gei84, Zum93] is displayed in Fig. 3.1. Here points are not restricted anymore onto the unit interval. Due to the coupling between different unit cells by eliminating the modulus, there are now 'jumps' possible from unit interval to unit interval. One may thus think of this dynamical system as a fully deterministic, anomalous version of a simple random walk on the line. A basic question is now which type of diffusion is generated by this model? As usual, the diffusive behavior is quantified by the mean square displacement (MSD) defined by

$$
\left\langle x^{2}\right\rangle:=\int d x x^{2} \rho_{n}(x),
$$

where $\left\langle x^{2}\right\rangle$ is the second moment of the position PDF $\rho_{n}(x)$ at time step $n$. Starting from a given initial PDF $\rho_{0}(x)$ at time step $n=0$, points, or point particles, will spread out over the whole real line, as quantified by $\rho_{n}(x)$. Surprisingly, by calculating this MSD both analytically and from computer simulations one finds [Gei84, Zum93] that for $z>2$

$$
\left\langle x^{2}\right\rangle \sim n^{\alpha} \quad, \quad \alpha<1 \quad(n \rightarrow \infty) .
$$

If one defines the diffusion coefficient of the system in the standard way by

$$
D:=\lim _{n \rightarrow \infty} \frac{\left\langle x^{2}\right\rangle}{2 n},
$$

Eq. (3.5) implies that $D=0$, despite the fact that particles can go anywhere on the real line as illustrated in Fig. 3.1. While a process like Brownian motion leads to $D>0$, here we thus encounter a very different type of diffusion: If the exponent $\alpha$ in the temporal spreading of the MSD Eq. (3.5) of an ensemble of particles is not equal to one, one speaks of anomalous diffusion [Met00, Kla08]. If $\alpha<1$ one says that there is subdiffusion, for $\alpha>1$ there is superdiffusion. In case of linear spreading with $\alpha=1$ one refers to normal diffusion. The constant

$$
K:=\lim _{n \rightarrow \infty} \frac{<x^{2}>}{n^{\alpha}},
$$




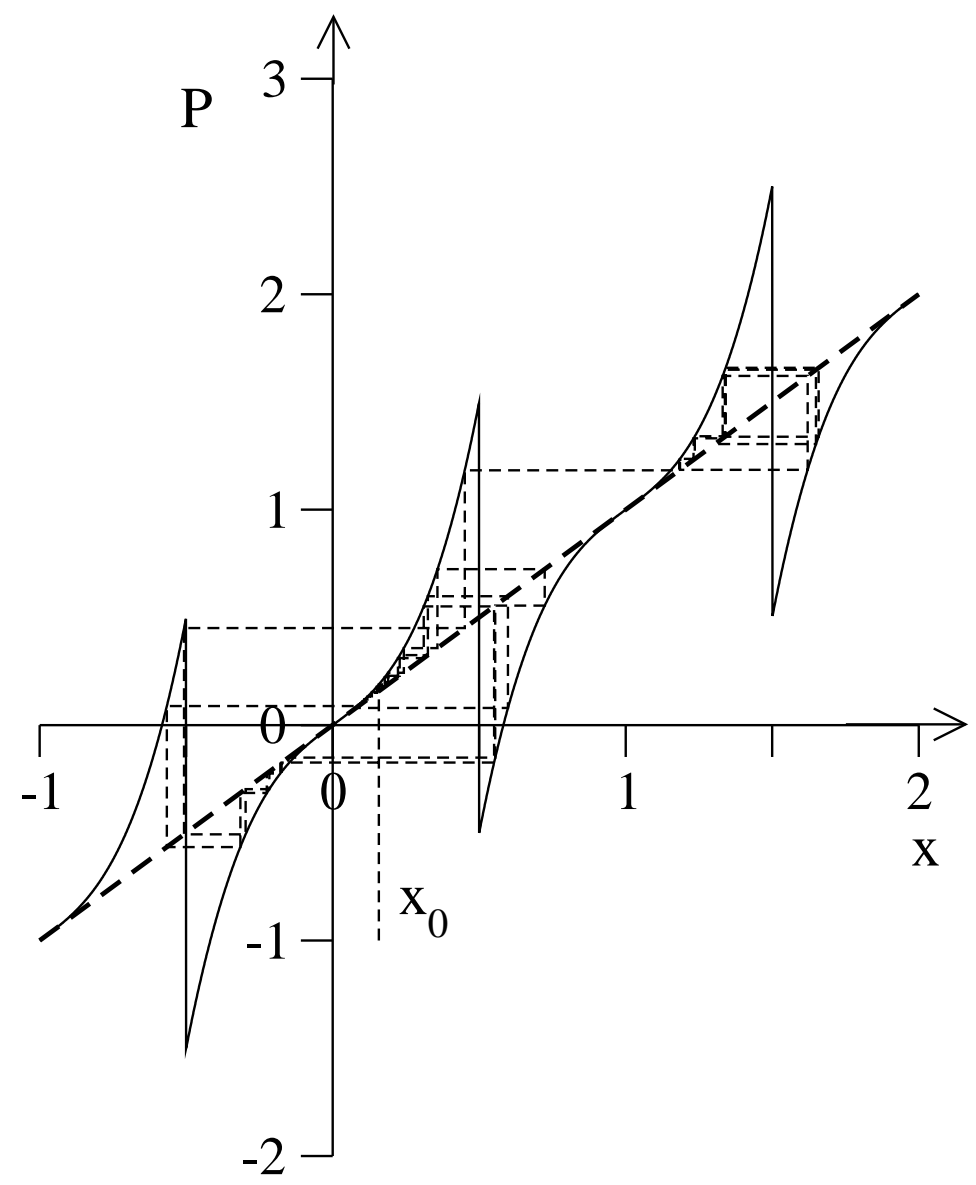

Figure 3.1: The Pomeau-Manneville map Fig. 2.2, Eq. (2.13), lifted symmetrically onto the whole real line such that it generates subdiffusion.

where in case of normal diffusion in one dimension $K=2 D$, is called the generalized diffusion coefficient. ${ }^{1}$ For our simple map model depicted in Fig. 3.1 we will first calculate the MSD analytically by means of stochstic continuous time random walk (CTRW) theory. By comparing the analytical formula with results from computer simulations, we will then focus on how $K$ behaves as a function of $z$ for fixed $a$ revealing some interesting, non-trivial properties.

\subsection{Continuous time random walk theory}

Pioneered by Montroll, Weiss and Scher [Mon65, Mon73, Sch75], CTRW theory yields perhaps the most fundamental theoretical approach to explain anomalous diffusion [Bou90, Wei94, Ebe05]. In further groundbreaking works by Geisel et al. and Klafter et al., this method was then adapted to sub- and superdiffusive deterministic maps [Gei84, Gei85, Sh185, Zum93]

The basic assumption of this approach is that diffusion can be decomposed into two stochastic processes characterized by waiting times and jumps, respectively. Thus one has two sequences of independent identically distributed random variables, namely a sequence of

\footnotetext{
${ }^{1}$ In detail, the definition of a generalized diffusion coefficient is a bit more subtle [Kor07].
} 
positive random waiting times $T_{1}, T_{2}, T_{3}, \ldots$ with PDF $w(t)$ and a sequence of random jumps $\zeta_{1}, \zeta_{2}, \zeta_{3}, \ldots$ with a PDF $\lambda(x)$. For example, if a particle starts at point $x=0$ at time $t_{0}=0$ and makes a jump of length $\zeta_{n}$ at time $t_{n}=T_{1}+T_{2}+\ldots+T_{n}$, its position is $x=0$ for $0 \leq t<T_{1}=t_{1}$ and $x=\zeta_{1}+\zeta_{2}+\ldots+\zeta_{n}$ for $t_{n} \leq t<t_{n+1}$. The probability that at least one jump is performed within the time interval $[0, t)$ is then $\int_{0}^{t} d t^{\prime} w\left(t^{\prime}\right)$ while the probability for no jump during this time interval reads $\Psi(t)=1-\int_{0}^{t} d t^{\prime} w\left(t^{\prime}\right)$. The master equation for the $\operatorname{PDF} P(x, t)$ to find a particle at position $x$ and time $t$ is then

$$
P(x, t)=\int_{-\infty}^{\infty} d x^{\prime} \lambda\left(x-x^{\prime}\right) \int_{0}^{t} d t^{\prime} w\left(t-t^{\prime}\right) P\left(x^{\prime}, t^{\prime}\right)+\Psi(t) \delta(x),
$$

which has the following probabilistic meaning: The PDF to find a particle at position $x$ at time $t$ is equal to the PDF to find it at point $x^{\prime}$ at some previous time $t^{\prime}$ multiplied with the transition probability to get from $\left(x^{\prime}, t^{\prime}\right)$ to $(x, t)$ integrated over all possible values of $x^{\prime}$ and $t^{\prime}$. The second term accounts for the probability of remaining at the initial position $x=0$. The most convenient representation of this equation is in Fourier-Laplace space,

$$
\hat{\tilde{P}}(k, s)=\int_{-\infty}^{\infty} d x e^{i k x} \int_{0}^{\infty} d t e^{-s t} P(x, t)
$$

where the hat stands for the Fourier transform and the tilde for the Laplace transform. Respectively, this function obeys the Fourier-Laplace transform of Eq. (3.8), which is called the Montroll-Weiss equation [Mon65, Mon73, Sch75],

$$
\hat{\tilde{P}}(k, s)=\frac{1-\tilde{w}(s)}{s} \frac{1}{1-\hat{\lambda}(k) \tilde{w}(s)} .
$$

The Laplace transform of the MSD can be obtained by differentiating the Fourier-Laplace transform of the PDF,

$$
\left.\left\langle x^{2} \tilde{(} s\right)\right\rangle=\int_{-\infty}^{\infty} d x x^{2} \tilde{P}(x, s)=-\left.\frac{\partial^{2} \hat{\tilde{P}}(k, s)}{\partial k^{2}}\right|_{k=0}
$$

In order to calculate the MSD within this theory, it thus suffices to know $\lambda(x)$ and $w(t)$ generating the stochastic process. For one-dimensional maps of the type of Eqs. (3.1),(3.2), by exploiting the symmetry of the map the waiting time distribution can be calculated from the approximation

$$
x_{n+1}-x_{n} \simeq \frac{d x_{t}}{d t}=a x_{t}^{z}, \quad x \ll 1,
$$

where we have introduced the continuous time $t \geq 0$. This equation can be solved for $x_{t}$ with respect to an initial condition $x_{0}$. Now one needs to define when a particle makes a "jump", as will be discussed below. By inverting the solution for $x_{t}$, one can then calculate the time $t$ a particle has to wait before it makes a jump as a function of the initial condition $x_{0}$. This information determines the relation between the waiting time PDF $w(t)$ and the as yet unknown PDF of injection points,

$$
w(t) \simeq P_{i n}\left(x_{0}\right)\left|\frac{d x_{0}}{d t}\right|
$$


Making the assumption that the PDF of injection points is uniform, $P_{\text {in }} \simeq 1$, the waiting time PDF is straightforwardly calculated from the knowledge of $t\left(x_{0}\right)$. The second ingredient that is needed for the CTRW approach is the jump PDF. Standard CTRW theory takes jumps between neighbouring cells only into account leading to the ansatz [Gei84, Zum93]

$$
\lambda(x)=\delta(|x|-1)
$$

It turns out that in order to qualitiatively reproduce the dependence of the generalized diffusion coefficient $K=K(z, a)$ Eq. (3.7) on the map's two control parameters $z$ and $a$, one needs to modify the standard theory at three points [Kor05, Kor07]: Firstly, the waiting time PDF must be calculated according to the unit interval $[0,1]$, not according to $[-0.5,0.5]$, which is an alternative but not appropriate choice [Kla96b, Kla97], yielding

$$
w(t)=a(1+a(z-1) t)^{-\frac{z}{z-1}} .
$$

However, this PDF also accounts for attempted jumps to another cell, since after a step the particle may stay in the same cell with a probability of $(1-p)$. The latter quantity is roughly determined by the size of the escape region $p=\left(1-2 x_{c}\right)$ with $x_{c}$ as a solution of the equation $x_{c}+a x_{c}^{z}=1$. We thus model this fact, secondly, by a jump length distribution in the form of

$$
\lambda(x)=\frac{p}{2} \delta(|x|-l)+(1-p) \delta(x) .
$$

Thirdly, in order to capture the dependence of $K$ on $z$ for fixed $a$, we define a typical jump length as

$$
l=\left\{\left|\left[M_{a, z}(x)\right]\right|\right\},
$$

where the square brackets stand for the floor function, which gives the coarse-grained displacement in units of elementary cells. The curly brackets denote both a time and ensemble average over points leaving a box. Note that for capturing the dependence of $K$ on $a$ for fixed $z$ a different definition of the jump length is appropriate [Kor05, Kor07]. Working out the modified CTRW approximation sketched above by taking these three details into account one obtains the result for the exponent $\alpha$ of the MSD, Eq. (3.5),

$$
\alpha= \begin{cases}1, & 1 \leq z<2 \\ \frac{1}{z-1}, & 2 \leq z\end{cases}
$$

which matches to the standard theory [Gei84, Zum93]. This result is in excellent agreement to simulations for a broad range of control parameters $z$ and $a$. Building on this result, the generalized diffusion coefficient can be calculated to

$$
K=p l^{2} \times \begin{cases}a^{\gamma} \sin (\pi \gamma) / \pi \gamma^{1+\gamma} & , \quad 0<\gamma<1 \\ a(1-1 / \gamma) & , \quad 1 \leq \gamma<\infty\end{cases}
$$

with $\gamma:=1 /(z-1)$, which for $z \geq 2$ is identical to $\alpha$ of Eq. (3.18). In Fig. 3.2 this analytical approximation for $K$ is compared with computer simulation results as a function of $z$ for fixed $a$. There is good qualitative agreement between theory and simulations for $z>2$, which converge asymptotically to each other for large $z$. For $z<2$ there is reasonable qualitative agreement, though quantitative deviations, for $z$ close to 2 while the theory does not work anymore for $z \rightarrow 0$, a problem that is discussed in Ref. [Kor07]. 


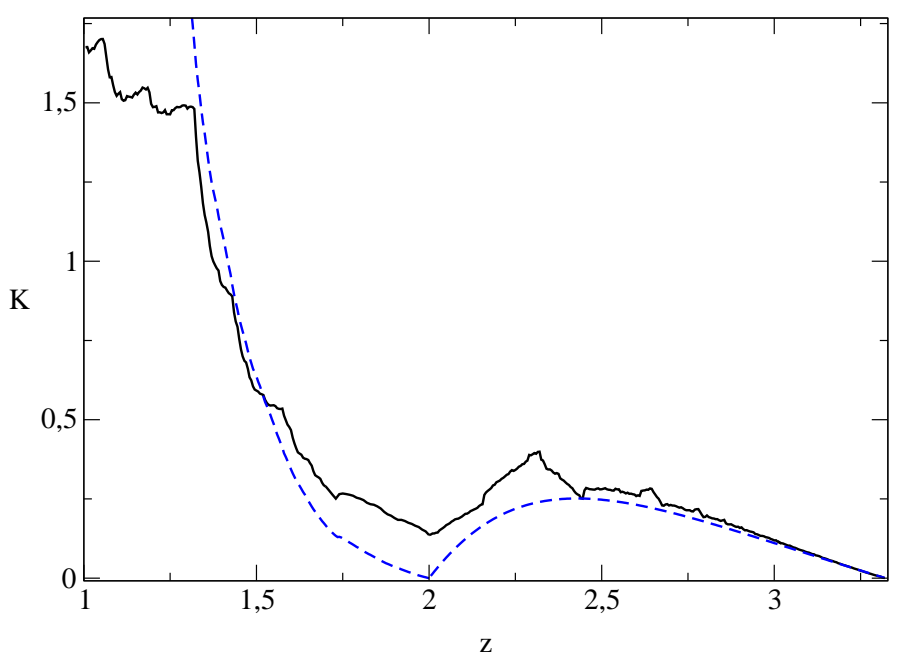

Figure 3.2: The generalized diffusion coefficient $K$, Eq. (3.7), for the spatially extended Pomeau-Manneville map displayed in Fig. 3.1 as a function of $z$ for $a=5$. The bold black line depicts computer simulation results. The dashed line corresponds to the modified CTRW approximation Eqs. (3.17),(3.19) [Kor07].

Remarkably, the $K(z)$ obtained from simulations does not appear to be a smooth function of the control parameter, which is at variance with the prediction of CTRW theory Eq. (3.19). This non-smooth parameter dependence is not due to numerical errors (which here are very difficult to assess, as discussed below) but a well-known phenomenon for this type of systems. It has first been discovered for the normal diffusive case of this map with $z=1$, where the diffusion coefficient $D=K / 2$ has been studied both numerically and analytically as a function of the slope $a$ as a control parameter [Kla95, Kla99]. Note that for $z=1$ the Pomeau-Manneville map boils down to a parameter-dependent, generalized version of the Bernoulli shift Eq. (2.2).

We do not wish to further elaborate on the fractal parameter dependencies of transport coefficients in simple deterministic dynamical systems, an interesting phenomenon that has been discussed in detail in Refs. [Kla96b, Kla07]. Rather, we focus on the behaviour of the generalized diffusion coefficient at the point $z=2$. According to the exponent $\alpha$ of the MSD given by Eq. (3.18), here the map exhibits a transition from normal to anomalous diffusion, which one may classify as a dynamical phase transition [Wan89, Bec93]. As can be seen in Fig. 3.2, right around this transition point there are significant deviations between CTRW theory and the simulation results. Most notably, at $z=2$ the CTRW approximation forms a non-differentiable little wedge by predicting $K(2)=0$, whereas the simulations yield $K(2)>0$. By increasing the computation time one indeed finds very slow convergence of the simulation data towards the CTRW solution [Kor07].

The explanation of these deviations, and of the phenomenon of a complete suppression of the generalized diffusion coefficient right at the transition point, is obtained by carrying out a refined analysis by means of CTRW theory. For a long time it was known already that at $z=2$, the MSD behaves like $\left\langle x^{2}\right\rangle \sim n / \ln n(n \rightarrow \infty)$ [Gei84, Zum93]. Note that according to our definition of the generalized diffusion coefficient Eq. (3.7) this logarithmic 
depencence was incorporated into the strength of the diffusion coefficient, otherwise our analytical CTRW approximation would not have been continuous at $z=2$. By taking into account higher-order terms when performing the CTRW theory calculations, which correspond to lower-order terms in time for the MSD, one arrives at

$$
\left\langle x^{2}\right\rangle \sim \begin{cases}\frac{n}{\ln n}, n<n_{c r} \text { and } \sim n, n \gg n_{c r}, & z<2 \\ \frac{n}{\ln n}, & z=2 \\ \frac{n^{\alpha}}{\ln n}, n<\tilde{n}_{c r} \text { and } \sim n^{\alpha}, n \gg \tilde{n}_{c r}, & z>2 .\end{cases}
$$

Here $n_{c r}$ and $\tilde{n}_{c r}$ are crossover times that can be calculated exactly in terms of the map's control parameters. For $z \rightarrow 2$ both these crossover times diverge, and one arrives at the asymptotic $n / \ln n$ dependence mentioned before. The perhaps surprising fact is that around the transition point these multiplicative logarithmic corrections still survive for long but finite time, in agreement with computer simulation results. In other words, these logarithmic corrections lead to an ultraslow convergence of the simulation results thus explaining the deviations between long-time CTRW theory and simulations shown in Fig. 3.2. But more importantly, these logarithmic terms dominate the strength of the generalized diffusion coefficient around the transition point from normal to anomalous diffusion eventually yielding a full suppression of this quantity right at the transition point. It can be conjectured that the presence of such multiplicative logarithmic corrections around transition points between normal and anomalous diffusion is a typical scenario in this type of systems [Kor07].

\subsection{A fractional diffusion equation}

We now turn to the PDFs generated by the lifted map Eq. (2.13). As we will show now, CTRW theory not only predicts the power $\alpha$ correctly but also the form of the coarse grained PDF $P(x, t)$ of displacements. Correspondingly the anomalous diffusion process generated by our model is not described by an ordinary diffusion equation but by a generalization of it. Starting from the Montroll-Weiss equation and making use of the expressions for the jump and waiting time distributions Eqs. (3.14), (3.15), we rewrite Eq. (3.10) in the long-time and -space asymptotic form

$$
s^{\gamma} \hat{\tilde{P}}-s^{\gamma-1}=-\frac{p l_{i}^{2}}{2 c b^{\gamma}} k^{2} \hat{\tilde{P}}
$$

with $c=\Gamma(1-\gamma)$ and $b=\gamma / a$. For the initial condition $P(x, 0)=\delta(x)$ of the PDF we have $\hat{P}(k, 0)=1$. Interestingly, the left hand side of this equation corresponds to the definition of the Caputo fractional derivative of a function $G$ [Pod99, Mai97],

$$
\frac{\partial^{\gamma} G}{\partial t^{\gamma}}:=\frac{1}{\Gamma(1-\gamma)} \int_{0}^{t} d t^{\prime}\left(t-t^{\prime}\right)^{-\gamma} \frac{\partial G}{\partial t^{\prime}},
$$

in Laplace space,

$$
\int_{0}^{\infty} d t e^{-s t} \frac{\partial^{\gamma} G}{\partial t^{\gamma}}=s^{\gamma} \tilde{G}(s)-s^{\gamma-1} G(0)
$$

Thus, fractional derivatives come naturally into play as a suitable mathematical formalism whenever there are power law memory kernels in space and/or time generating anomalous dynamics; see, e.g., Refs. [Sok02, Met00] for short introductions to fractional derivatives 


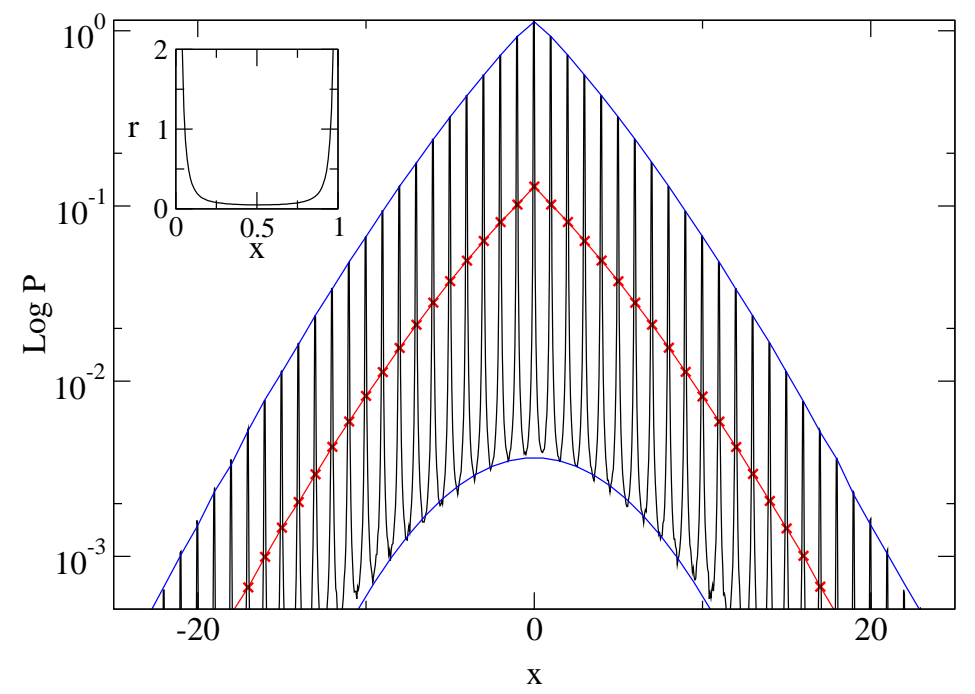

Figure 3.3: Comparison of the probability density obtained from simulations of the lifted map Eq. (2.13) (oscillatory structure) with the analytical solution Eq. (3.25) of the fractional diffusion equation Eq. (3.24) (continuous line in the middle) for $z=3$ and $a=8$. The probability density was computed from $10^{7}$ particles after $n=10^{3}$ iterations. For the generalized diffusion coefficient in Eq. (3.25) the simulation result was used. The crosses (x) represent the numerical results coarse grained over unit intervals. The upper and the lower curves correspond to fits with a stretched exponential and a Gaussian distribution, respectively. The inset depicts the probability density function for the map on the unit interval with periodic boundaries.

and Ref. [Pod99] for a detailed exposition. Turning back now to real space, we thus arrive at the time-fractional diffusion equation

$$
\frac{\partial^{\gamma} P(x, t)}{\partial t^{\gamma}}=D \frac{\partial^{2} P}{\partial x^{2}}
$$

with $D=K \Gamma(1+\gamma) / 2,0<\gamma<1$, which is an example of a fractional diffusion equation generating subdiffusion. Note the existence of the gamma function in the numerator defining $D$, which is analogous to the appearance of the gamma function in our generalized chaos quantities Eqs. (2.23),(2.24). For $\gamma=1$ we recover the ordinary diffusion equation. The solution of Eq. (3.24) can be expressed in terms of an M-function of Wright type [Mai97] and reads

$$
P(x, t)=\frac{1}{2 \sqrt{D} t^{\gamma / 2}} M\left(\xi, \frac{\gamma}{2}\right)
$$

Fig. 3.3 demonstrates an excellent agreement between the analytical solution Eq. (3.25) and the PDF obtained from simulations of the map Eq. (2.13) if it is coarse grained over unit intervals. However, it also shows that the coarse graining eliminates a periodic fine structure that is not captured by Eq. (3.25). This fine structure derives from the 'microscopic' invariant density of an elementary cell (with periodic boundaries) as shown in the inset of Fig. 3.3 [Kla96b]. The singularities are due to the marginal fixed points of the map, where points are trapped for long times. Remarkably, that way the microscopic origin of the intermittent dynamics is reflected in the shape of the PDF on the whole real line: From Fig. 3.3 it is seen that the oscillations in the density are bounded by two functions, the upper curve being of 
a stretched exponential type while the lower is Gaussian. These two envelopes correspond to the laminar and chaotic parts of the motion, respectively.

\section{Anomalous fluctuation relations}

After having accomplished a transition from deterministic dynamics to stochastic modeling in the previous section, for the reminder of this chapter we fully focus on stochastic systems. First, we discuss a remarkable finding in nonequilibrium statistical mechanics that was widely investigated over the past two decades, which are fluctuation relations. After providing a brief outline of what they are, and why they are important, we first study an example of them for one of the most simple types of stochastic dynamics, which is Brownian motion modeled by an ordinary Langevin equation. Along these lines, we then consider generalized versions of Langevin dynamics exhibiting anomalous diffusion. For these types of dynamics we check again for fluctuation relations and in one case obtain a different, new form of such a formula. We argue that generalized, anomalous fluctuation relations should be important to understand nonequilibrium fluctuations in glassy dynamics.

\subsection{Fluctuation relations}

Fluctuation Relations (FRs) denote a set of symmetry relations describing large-deviation properties of the PDFs of statistical physical observables far from equilibrium. First forms defining one subset of them, often referred to as Fluctuation Theorems, emerged from generalizing fluctuation-dissipation relations to nonlinear stochastic processes [Boc81a, Boc81b]. They were then discovered as generalizations of the Second Law of Thermodynamics for thermostated dynamical systems, i.e., systems interacting with thermal reservoirs, in nonequilibrium steady states [Eva93, Eva94, Gal95a, Ga195b]. Another subset, called work relations, generalize a relation between work and free energy, known from equilibrium thermodynamics, to nonequilibrium situations [Jar97b, Jar97a]. These two fundamental classes were later on amended and generalized by a variety of other FRs from which they can partially be derived as special cases [Cro99, Hat01, Sei05, Sag10]. Research performed over the past ten years has shown that FRs hold for a great variety of systems thus featuring one of the rare statistical physical principles that is valid even very far from equilibrium; see, e.g., Refs. [Ga198, Eva02, Kla07, Har07, Sei08, Jar08, Kla12b] and further references therein. Many of these relations have meanwhile been verified in experiments on small systems, i.e., systems on molecular scales featuring only a limited number of relevant degrees of freedom [Wan02, Rit03, Bus05, Cil10, Toy10, Ale11].

So far FRs have mostly been studied for dynamics exhibiting normal diffusion. This raises the question to which extent the 'conventional' FRs derived for these cases are valid for anomalous dynamics [Che09, Kla12a]. Theoretical results for generalized Langevin equations [Bec04, Ohk07, Mai07, Cha08], Lévy flights [Tou07, Tou09] and continuous-time random walk models [Esp08] as well as computer simulations for glassy dynamics [Sel09] showed 


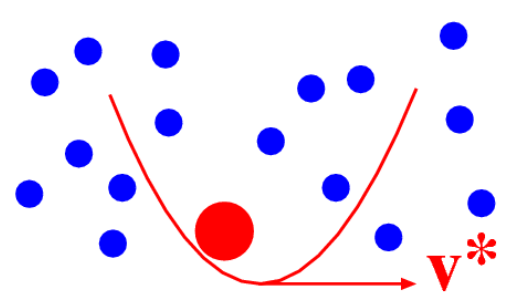

Figure 4.1: Sketch of a colloidal particle confined within a harmonic trap that is dragged through water with a constant velocity $v^{*}$, cf. the experiment by Wang et al. [Wan02].

both validity and violations of the various types of conventional FRs referred to above, depending on the specific type of anomalous dynamics considered and the nonequilibrium conditions that have been applied [Che09].

In this section we outline how the two different fields of FRs and anomalous dynamics can be cross-linked in order to explore to which extent conventional forms of FRs are valid for anomalous dynamics. With the term anomalous fluctuation relations we thus refer to deviations from conventional forms of FRs, which are due to anomalous dynamics. Here we focus on generic types of stochastic anomalous dynamics by only checking transient fluctuation relations (TFRs), which describe the approach from a given initial distribution towards a (non)equilibrium steady state. As a warm-up, we first derive the conventional TFR for the trivial case of Brownian motion of a particle moving under a constant external force modeled by standard Langevin dynamics. We then consider a straightforward generalization of this type of dynamics in form of long-time correlated Gaussian stochastic processes. For two fundamental, different versions of this dynamics we check for the existence of conventional TFRs under the simple nonequilibrium condition of a constant external force.

\subsection{Fluctuation relations for ordinary Langevin dynamics}

Consider a particle system evolving from some initial state at time $t=0$ into a nonequilibrium steady state for $t \rightarrow \infty$. A famous example that has been investigated experimentally [Wan02] is a colloidal particle immersed into water and confined by an optical harmonic trap, see Fig. 4.1. The trap is first at rest but then dragged through water with a constant velocity $v^{*}$.

The key for obtaining FRs in such systems is to compute the PDF $\rho\left(\xi_{t}\right)$ of suitably defined dimensionless entropy production $\xi_{t}$ over trajectory segments of time length $t$. The goal is to quantify the asymmetry between positive and negative entropy production in $\rho\left(\xi_{t}\right)$ for different times $t$ since, as we will demonstrate in a moment, this relation is intimately related to the Second Law of Thermodynamics. For a very large class of systems, and under rather general conditions, it was shown that the following equation holds [Eva02, Har07, Kur07]:

$$
\ln \frac{\rho\left(\xi_{t}\right)}{\rho\left(-\xi_{t}\right)}=\xi_{t}
$$

Given that here we consider the transient evolution of a system from an initial into a steady state, this formula became known as the transient fluctuation relation (TFR). The left hand side we may call the fluctuation ratio. Relations exhibiting this functional form have first been proposed in the seminal work by Evans, Cohen and Morriss [Eva93], although in the 


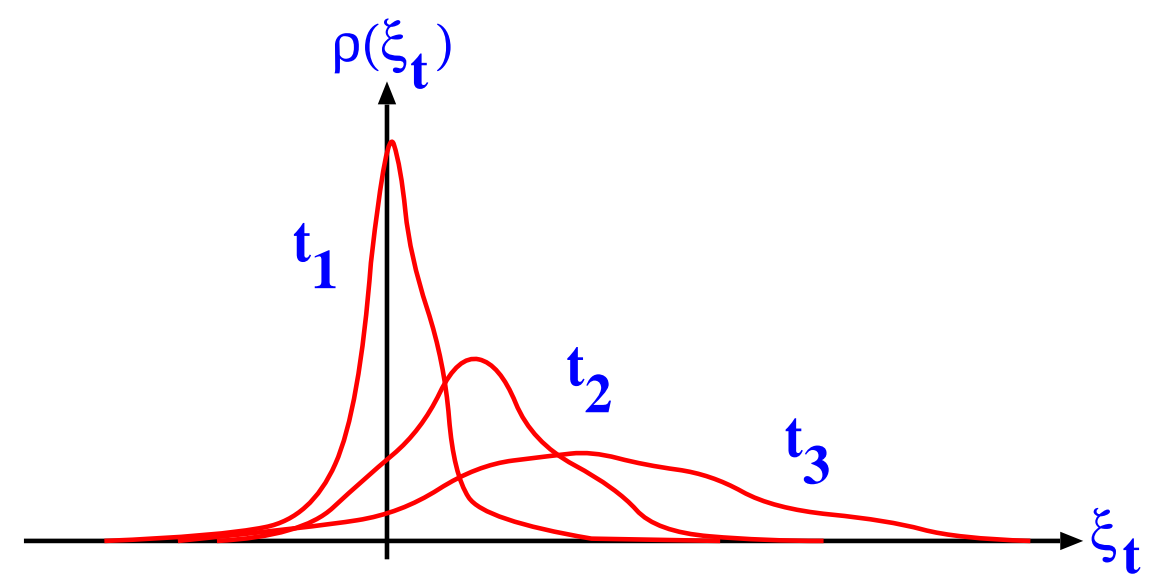

Figure 4.2: Illustration of the dynamics of the probability density function for entropy production $\rho\left(\xi_{t}\right)$ for different times $t_{1}<t_{2}<t_{3}$.

different situation of considering nonequilibrium steady states. Such a steady state relation was proved a few years later on by Gallavotti and Cohen for deterministic dynamical systems, based on the chaotic hypothesis [Ga195a, Gal95b]. The idea to consider such relations for transient dynamics was first put forward by Evans and Searles [Eva94].

Fig. 4.2 displays the temporal evolution of the PDF for entropy production in such a situation. The asymmetry of the evolving distribution, formalized by the fluctuation relation Eq. (4.1), is in line with the Second Law of Thermodynamics. This easily follows from Eq. (4.1) by noting that

$$
\rho\left(\xi_{t}\right)=\rho\left(-\xi_{t}\right) \exp \left(\xi_{t}\right) \geq \rho\left(-\xi_{t}\right),
$$

where $\xi_{t}$ is taken to be positive or zero. Integration from zero to infinity over both sides of this inequality after multiplication with $\xi_{t}$ and defining the ensemble average over the given $\mathrm{PDF}$ as $\langle\ldots\rangle=\int_{-\infty}^{\infty} d \xi_{t} \rho\left(\xi_{t}\right) \ldots$ yields

$$
\left\langle\xi_{t}\right\rangle \geq 0 .
$$

As a warm-up, we may first check the TFR for the ordinary overdamped Langevin equation [Kub92]

$$
\dot{x}=F+\zeta(t),
$$

with a constant external force given by $F$ and Gaussian white noise $\zeta(t)$. Note that for sake of simplicity, here we set all the other constants that are not relevant within this specific context equal to one. For Langevin dynamics with a constant force the entropy production $\xi_{t}$ defined by the heat, or equivalently the dissipative work, is simply equal to the mechanical work [vZ03b]

$$
W_{t}=F x(t) .
$$

It follows that the PDF of entropy production, which here is identical to the one for the mechanical work, is trivially related to the PDF of the position $x$ of the Langevin particle via

$$
\rho\left(W_{t}\right)=F^{-1} \varrho(x, t) .
$$

This is very convenient, since it implies that all that remains to be done in order to check the TFR Eq. (4.1) is to solve the Fokker-Planck equation for the position PDF $\varrho(x, t)$ for 
a given initial condition. Here and in the following, we choose $x(0)=0$, i.e., in terms of position PDFs we start with a delta-distribution at $x=0$. Note that for ordinary Langevin dynamics in a given potential, typically the equilibrium density is taken as the initial density [vZ03a, vZ03b]. However, since in the following we will consider dynamics that may not exhibit a simple equilibrium state, without loss of generality here we make a different choice. For the ordinary Langevin dynamics Eq. (4.4) modeling a linear Gaussian stochastic process, the position PDF is Gaussian exhibiting normal diffusion [Kub92, Ris96],

$$
\varrho(x, t)=\frac{1}{\sqrt{2 \pi \sigma_{x, 0}^{2}}} \exp \left(-\frac{(x-\langle x\rangle)^{2}}{2 \sigma_{x, 0}^{2}}\right) .
$$

With the subscript zero we denote ensemble averages in case of zero external field. By using the PDF-scaling Eq. (4.6) and plugging this result into the TFR Eq. (4.1), we easily derive that the TFR for the work $W_{t}$ holds if

$$
\left\langle W_{t}\right\rangle=\frac{\sigma_{W_{t}, 0}^{2}}{2},
$$

which is nothing else than an example of the fluctuation-dissipation relation of the first kind (FDR1) [Kub66, Kub92]. We thus arrive at the seemingly trivial but nevertheless important result that for this simple Gaussian stochastic process, the validity of FDR1 Eq. (4.8) implies the validity of the work TFR Eq. (4.1). For a full analysis of FRs of ordinary Langevin dynamics we refer to van Zon and Cohen Refs. [vZ03a, vZ03b]. Probably inspired by the experiment of Ref. [Wan02], typically Langevin dynamics in a harmonic potential moving with a constant velocity has been studied in the literature [Zam05a, Mai07, Ohk07, Cha08], cf. Fig. 4.1. Note that in this slightly more complicated case the (total) work is not equal to the heat [vZ03b]. While for the work one recovers the TFR in its conventional form Eq. (4.1) in analogy to the calculation above, surprisingly the TFR for heat looks different for large enough fluctuations. The origin of this phenomenon has been discussed in detail in Ref. [vZ03a], related effects have been reported in Refs. [Har06, Eva05, Har07]. However, in the following we check for another source of deviations from the conventional TFR Eq. (4.1) that is due to the existence of microscopic correlations in form of anomalous dynamics. In order to illustrate the main ideas it suffices to consider a nonequilibrium situation simply generated by a constant external force.

\subsection{Fluctuation relations for anomalous Langevin dynamics}

In our presentation of this section we follow Ref. [Che09], which may be consulted for further details. Our goal is to check the TFR Eq. (4.1) for Gaussian stochastic processes generating anomalous diffusion. These processes are defined by the overdamped generalized Langevin equation

$$
\int_{0}^{t} d t^{\prime} \dot{x}\left(t^{\prime}\right) \gamma\left(t-t^{\prime}\right)=F+\zeta(t)
$$

with Gaussian noise $\zeta(t)$ and friction that is modeled with a memory kernel $\gamma(t)$. By using this equation a stochastic process can be defined that exhibits normal statistics but 
with anomalous memory properties in form of non-Markovian long-time correlated Gaussian noise. Equations of this type can be traced back at least to work by Mori and Kubo around 1965 (see [Kub66] and further references therein). They form a class of standard models generating anomalous diffusion that has been widely investigated, see, e.g., Refs. [Kub92, Por96, Lut01]. FRs for this type of dynamics have more recently been analyzed in Refs. [Bec04, Ohk07, Mai07, Cha08]. Examples of applications for this type of stochastic modeling are given by generalized elastic models [Tal10], polymer dynamics [Pan10] and biological cell migration [Die08]. We split this class into two specific cases:

\section{Correlated internal Gaussian noise}

We speak of internal Gaussian noise in the sense that we require the system to exhibit the fluctuation-dissipation relation of the second kind (FDR2) [Kub66, Kub92]

$$
\left\langle\zeta(t) \zeta\left(t^{\prime}\right)\right\rangle \sim \gamma\left(t-t^{\prime}\right)
$$

again by neglecting all constants that are not relevant for the main point we wish to make here. We now consider the specific case that both the noise and the friction are correlated by a simple power law,

$$
\gamma(t) \sim t^{-\beta}, 0<\beta<1
$$

Because of the linearity of the generalized Langevin equation (4.9) the position PDF must be the Gaussian Eq. (4.7), and by the scaling of Eq. (4.6) we have $\rho\left(W_{t}\right) \sim \varrho(x, t)$. It thus remains to solve Eq. (4.9) for mean and variance, which can be done in Laplace space [Che09] yielding subdiffusion,

$$
\sigma_{x, F}^{2} \sim t^{\beta}
$$

by preserving the FDR1 Eq. (4.8). Here and in the following we denote ensemble averages in case of a non-zero external field with the subscript $F$. For Gaussian stochastic processes we have seen in the previous section that the conventional work TFR follows from FDR1. Hence, for the above power-law correlated internal Gaussian noise we recover the conventional work TFR Eq. (4.1).

\section{Correlated external Gaussian noise}

As a second case, we consider the overdamped generalized Langevin equation

$$
\dot{x}=F+\zeta(t)
$$

which represents a special case of Eq. (4.9) with a memory kernel modeled by a deltafunction. Again we use correlated Gaussian noise defined by the power law

$$
\left\langle\zeta(t) \zeta\left(t^{\prime}\right)\right\rangle \sim\left|t-t^{\prime}\right|^{-\beta}, 0<\beta<1,
$$

which one may call external, because in this case we do not postulate the existence of FDR2. The position PDF is again Gaussian, and as before $\rho\left(W_{t}\right) \sim \varrho(x, t)$. However, by solving the Langevin equation along the same lines as in the previous case, here one obtains superdiffusion by breaking FDR1,

$$
\left\langle W_{t}\right\rangle \sim t \quad, \quad \sigma_{W_{t}, F}^{2} \sim t^{2-\beta} .
$$




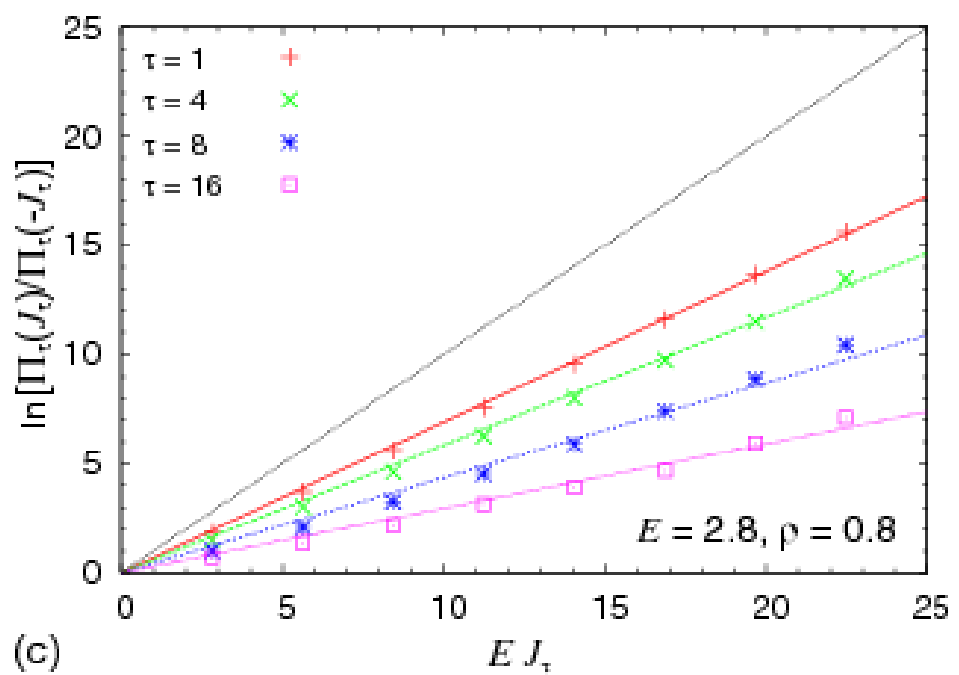

Figure 4.3: The fluctuation ratio $\ln \left(\Pi_{\tau}\left(J_{\tau}\right) / \Pi_{\tau}\left(J_{\tau}\right)\right)$ for the entropy production $W_{\tau}=E J_{\tau}$ with particle current $J_{\tau}$ and field strength $E$ for particle density $\rho$ at different times $\tau$. The full line, with slope one, displays the result of the conventional FR Eq. (4.1) in a nonequilibrium steady state. The figure is from Ref. [Sel09].

Calculating the fluctuation ratio, i.e., the left hand side of Eq. (4.1), from these results yields the anomalous work TFR

$$
\ln \frac{\rho\left(W_{t}\right)}{\rho\left(-W_{t}\right)}=C_{\beta} t^{\beta-1} W_{t} \quad 0<\beta<1,
$$

where $C_{\beta}$ is a constant that depends on physical parameters [Che09]. Comparing this equation with the conventional form of the TFR Eq. (4.1) one observes that the fluctuation ratio is still linear in $W_{t}$ thus exhibiting an exponential large deviation form [Tou09]. However, there are two important deviations: (1) the slope of the fluctuation ratio as a function of $W_{t}$ is not equal to one anymore, and in particular (2) it decreases with time. We may thus classify Eq. (4.16) as a weak violation of the conventional TFR.

We remark that for driven glassy systems FRs have already been obtained displaying slopes that are not equal to one. Within this context it has been suggested to capture these deviations from one by introducing the concept of an 'effective temperature' [Sel98, Zam05b, Zam05a]. As far as the time dependence of the coefficient is concerned, such behavior has recently been observed in computer simulations of a paradigmatic two dimensional lattice gas model generating glassy dynamics [Sel09]. Fig. 4.3 shows the fluctuation ratio as a function of the entropy production at different times $\tau$ as extracted from computer simulations of this model, where the PDF has first been relaxed into a nonequilibrium steady state. It is clearly seen that the slope decreases with time, which is in line with the prediction of the anomalous TFR Eq. (4.16). To which extent the nonequilibrium dynamics of this lattice gas model can be mapped onto the generalized Langevin equation Eq. (4.13) is an open question.

In summary, for Gaussian stochastic processes with correlated noise the existence of FDR2 implies the existence of FDR1, and FDR1 in turn implies the existence of work TFR in conventional form. That is, the conventional work TFR holds for internal noise. However, 
there is a weak violation of the conventional form in case of external noise yielding a prefactor that is not equal to one and in particular depends on time.

\section{Anomalous dynamics of biological cell migration}

In order to illustrate the importance of anomalous dynamics for realistic situations, in this final section of our book chapter we discuss experiments and theory about the migration of single biological cells crawling on surfaces as an example. Here we focus on cells in an equilibrium situation, i.e., not moving under the influence of any external gradients or fields. This case is investigated by extracting results for the MSD and for the position PDFs from experimental data. We then show how the experimental results can be understood by a mathematical model in form of a fractional Klein-Kramers equation. As far as MSD and velocity autocorrelation function are concerned, this equation bears some similarity to a generalized Langevin equation that is of the same type as the one that has been discussed in Section 4.3. Our presentation in this section is based on Ref. [Die08].

\subsection{Cell migration}

Nearly all cells in the human body are mobile at a given time during their life cycle. Embryogenesis, wound-healing, immune defense and the formation of tumor metastases are well known phenomena that rely on cell migration [Lau96, Läm09, Fri10]. Fig. 5.1 depicts the path of a single biological cell crawling on a substrate measured in an in vitro experiment [Die08]. At first sight, the path looks like the trajectory of a Brownian particle generated, e.g., by the ordinary Langevin dynamics of Eq. (4.4). On the other hand, according to Einstein's theory of Brownian motion a Brownian particle is passively driven by collisions from the surrounding fluid molecules, whereas biological cells move actively by themselves converting chemical into kinetic energy. This raises the question whether the random-looking paths of crawling biological cells can really be understood in terms of simple Brownian motion [Dun87, Sto91] or whether more advanced concepts of dynamical modeling have to be applied [Har94, Upa01, Li08, Tak08, Böd10].

\subsection{Experimental results and statistical analysis}

The cell migration experiments that we now discuss have been performed on two types of tumor-like migrating transformed renal epithelial Madin Darby canine kidney (MDCK-F) cell strains: wild-type $\left(\mathrm{NHE}^{+}\right)$and NHE-deficient $\left(\mathrm{NHE}^{-}\right)$cells. Here $\mathrm{NHE}^{+}$stands for a molecular sodium hydrogen exchanger that either is present or deficient. It can thus be checked whether this microscopic exchanger has an influence on cell migration, which 


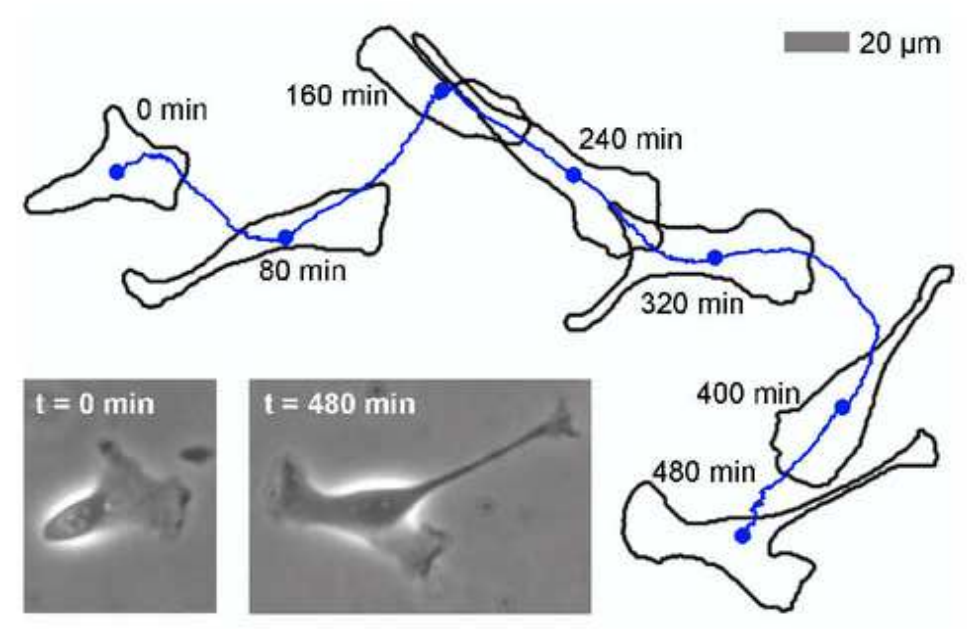

Figure 5.1: Overlay of a biological cell migrating in vitro on a substrate. The cell frequently changes its shape and direction during migration, as is shown by several cell contours extracted during the migration process. The inset displays phase contrast images of the cell at the beginning and to the end of its migration process [Die08].

is a typical question asked by cell physiologists. The cell diameter is about $20-50 \mu \mathrm{m}$ and the mean velocity of the cells about $1 \mu \mathrm{m} / \mathrm{min}$. Cells are driven by active protrusions of growing actin filaments (lamellipodial dynamics) and coordinated interactions with myosin motors and dynamically re-organizing cell-substrate contacts. The leading edge dynamics of a polarized cell proceeds at the order of seconds. Thirteen cells were observed for up to 1000 minutes. Sequences of microscopic phase contrast images were taken and segmented to obtain the cell boundaries shown in Fig. 5.1; see Ref. [Die08] for full details of the experiments.

According to the Langevin description of Brownian motion outlined in Section 4.2, Brownian motion is characterized by a MSD $\sigma_{x, 0}^{2}(t) \sim t(t \rightarrow \infty)$ designating normal diffusion. Fig. 5.2 shows that both types of cells behave differently: First of all, MDCK-F $N H E^{-}$cells move less efficiently than $\mathrm{NHE}^{+}$cells resulting in a reduced MSD for all times. As is displayed in the upper part of this figure, the MSD of both cell types exhibits a crossover between three different dynamical regimes. These three phases can be best identified by extracting the time-dependent exponent $\beta$ of the MSD $\sigma_{x, 0}^{2}(t) \sim t^{\beta}$ from the data, which can be done by using the logarithmic derivative

$$
\beta(t)=\frac{d \ln m s d(t)}{d \ln t} .
$$

The results are shown in the lower part of Fig. 5.2. Phase I is characterized by an exponent $\beta(t)$ roughly below 1.8. In the subsequent intermediate phase II, the MSD reaches its strongest increase with a maximum exponent $\beta$. When the cell has approximately moved beyond a square distance larger than its own mean square radius (indicated by arrows in the figure), $\beta(t)$ gradually decreases to about 1.4. Both cell types therefore do not exhibit normal diffusion, which would be characterized by $\beta(t) \rightarrow 1$ in the long time limit, but move anomalously, where the exponent $\beta>1$ indicates superdiffusion.

We next study the PDF of cell positions. Since no correlations between $x$ and $y$ positions 

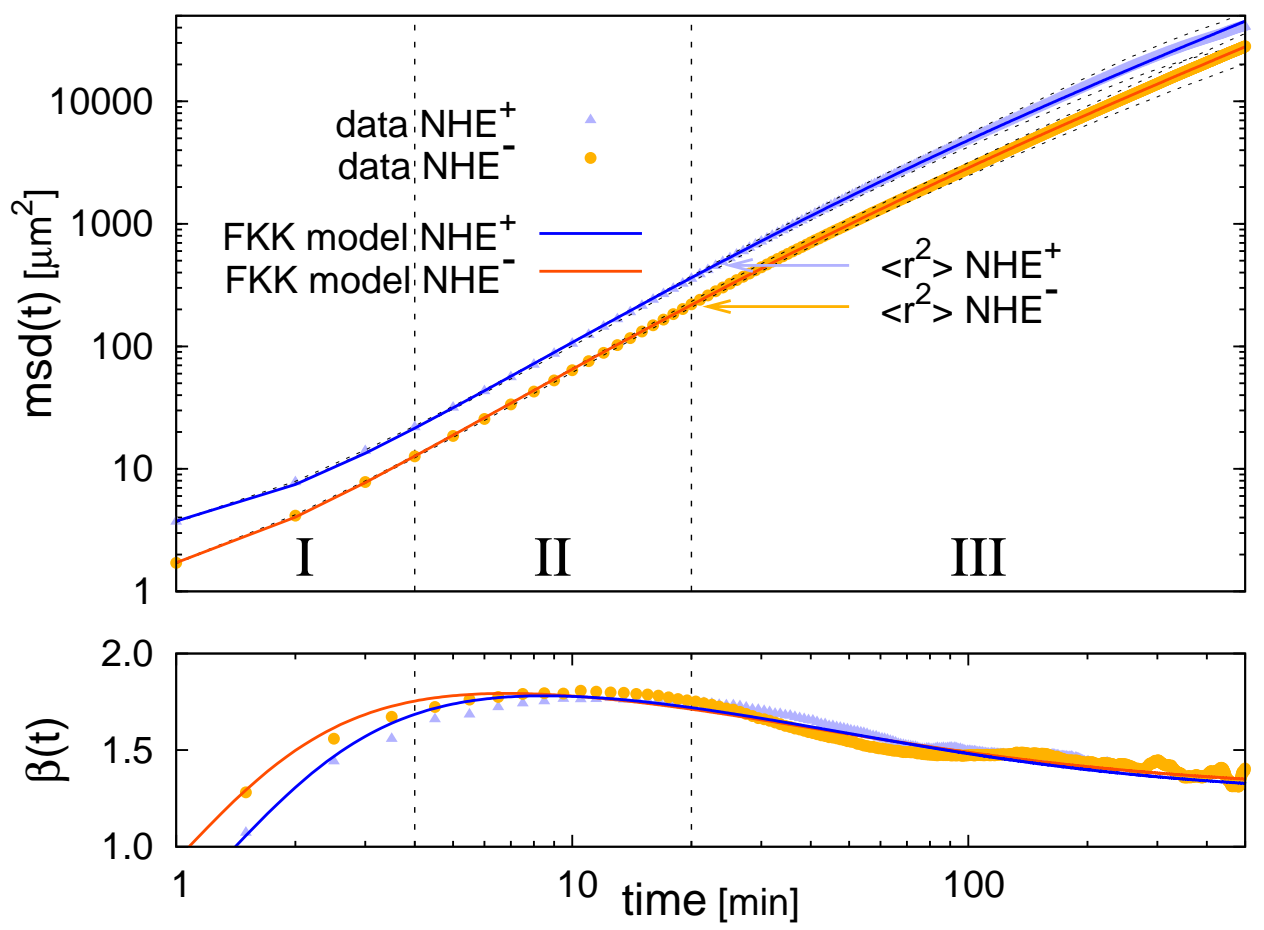

Figure 5.2: Upper part: Double-logarithmic plot of the mean square displacement (MSD) as a function of time. Experimental data points for both cell types are shown by symbols. Different time scales are marked as phases I, II and III as discussed in the text. The solid lines represent fits to the MSD from the solution of our model, see Eq. (5.7). All parameter values of the model are given in [Die08]. The dashed lines indicate the uncertainties of the MSD values according to Bayes data analysis. Lower part: Logarithmic derivative $\beta(t)$ of the MSD for both cell types as defined by Eq. (5.1).

could be found, it suffices to restrict ourselves to one dimension. Fig. 5.3 (a), (b) reveals the existence of non-Gaussian distributions at different times. The transition from a peaked distribution at short times to rather broad distributions at long times suggests again the existence of distinct dynamical processes acting on different time scales. The shape of these distributions can be quantified by calculating the kurtosis

$$
\kappa(t):=\frac{\left\langle x^{4}(t)\right\rangle}{\left\langle x^{2}(t)\right\rangle^{2}}
$$

which is displayed as a function of time in Fig. 5.3 (c). For both cell types $\kappa(t)$ rapidly decays to a constant that is clearly below three in the long time limit. A value of three would be the result for the spreading Gaussian distributions characterizing Brownian motion. These findings are another strong manifestation of the anomalous nature of cell migration.

\subsection{Stochastic modeling}

We now present the stochastic model that we have used to reproduce the experimental data yielding the fit functions shown in the previous two figures. The model is defined by the 

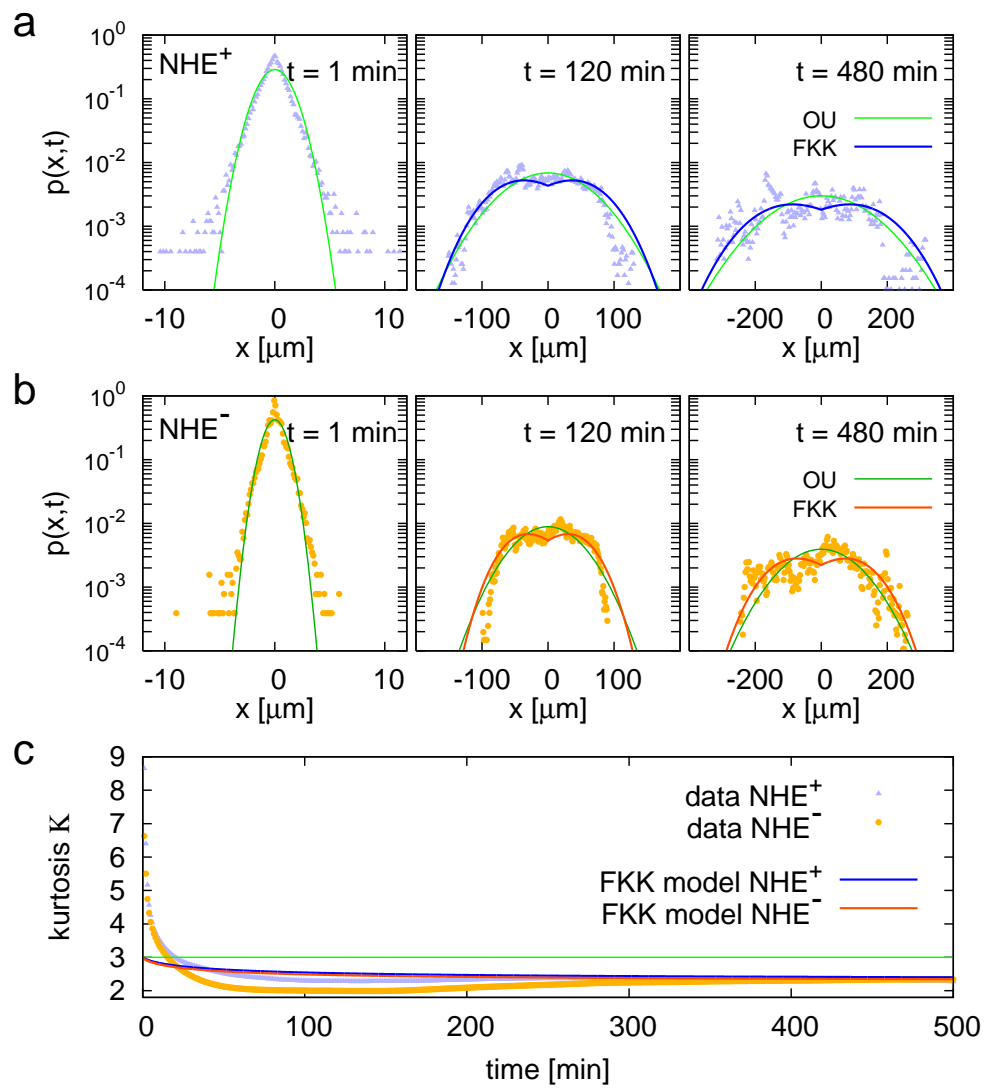

Figure 5.3: Spatio-temporal probability distributions $P(x, t)$. (a),(b): Experimental data for both cell types at different times in semilogarithmic representation. The dark lines, labeled FKK, show the long-time asymptotic solutions of our model Eq. (5.3) with the same parameter set used for the MSD fit. The light lines, labeled OU, depict fits by the Gaussian distributions Eq. (4.7) representing Brownian motion. For $t=1 \mathrm{~min}$ both $P(x, t)$ show a peaked structure clearly deviating from a Gaussian form. (c) The kurtosis $\kappa(t)$ of $P(x, t)$, cf. Eq. (5.2), plotted as a function of time saturates at a value different from the one of Brownian motion (line at $\kappa=3$ ). The other two lines represent $\kappa(t)$ obtained from the model Eq. (5.3) [Die08].

fractional Klein-Kramers equation [Bar00]

$$
\frac{\partial \varrho}{\partial t}=-\frac{\partial}{\partial x}[v \varrho]+\frac{\partial^{1-\alpha}}{\partial t^{1-\alpha}} \gamma_{\alpha}\left[\frac{\partial}{\partial v} v+v_{t h}^{2} \frac{\partial^{2}}{\partial v^{2}}\right] \varrho \quad, 0<\alpha<1 .
$$

Here $\varrho=\varrho(x, v, t)$ is the PDF depending on time $t$, position $x$ and velocity $v$ in one dimension, $\gamma_{\alpha}$ is a friction term and $v_{t h}^{2}=k_{B} T / M$ stands for the thermal velocity squared of a particle of mass $M=1$ at temperature $T$, where $k_{B}$ is Boltzmann's constant. The last term in this equation models diffusion in velocity space. In contrast to Fokker-Planck equations, this equation features time evolution both in position and velocity space. What distinguishes this equation from an ordinary Klein-Kramers equation, the most general model of Brownian motion [Ris96], is the presence of the Riemann-Liouville fractional derivative of order $1-\alpha$

$$
\frac{\partial^{1-\alpha}}{\partial t^{1-\alpha}} \varrho=\frac{\partial}{\partial t}\left[\frac{1}{\Gamma(\alpha)} \int_{0}^{t} d t^{\prime} \frac{\varrho\left(t^{\prime}\right)}{\left(t-t^{\prime}\right)^{1-\alpha}}\right]
$$


in front of the terms in square brackets. Note that for $\alpha=1$ the ordinary Klein-Kramers equation is recovered. The analytical solution of this equation for the MSD has been calculated in Ref. [Bar00] to

$$
\sigma_{x, 0}^{2}(t)=2 v_{t h}^{2} t^{2} E_{\alpha, 3}\left(-\gamma_{\alpha} t^{\alpha}\right) \quad \rightarrow \quad 2 \frac{D_{\alpha} t^{2-\alpha}}{\Gamma(3-\alpha)} \quad(t \rightarrow \infty)
$$

with $D_{\alpha}=v_{t h}^{2} / \gamma_{\alpha}$ and the two-parametric or generalized Mittag-Leffler function (see, e.g., Chapter 4 of Ref. [Kla08] and Refs. [Gor97, Pod99])

$$
E_{\alpha, \beta}(z)=\sum_{k=0}^{\infty} \frac{z^{k}}{\Gamma(\alpha k+\beta)}, \alpha, \beta>0, z \in \mathbb{C}
$$

Note that $E_{1,1}(z)=\exp (z)$, hence $E_{\alpha, \beta}(z)$ is a generalized exponential function. We see that for long times Eq. (5.5) yields a power law, which reduces to the long-time Brownian motion result in case of $\alpha=1$.

In view of the experimental data shown in Fig. 5.2, Eq. (5.5) was amended by including the impact of random perturbations acting on very short time scales for which we take Gaussian white noise of variance $\eta^{2}$. This leads to [Mar02]

$$
\sigma_{x, 0 ; \text { noise }}^{2}(t)=\sigma_{x, 0}^{2}(t)+2 \eta^{2}
$$

The second term mimicks both measurement errors and fluctuations of the cell cytoskeleton. In case of the experiments with MDCK-F cells [Die08], the value of $\eta$ can be extracted from the experimental data and is larger than the estimated measurement error. Hence, this noise must largely be of a biological nature and may be understood as being generated by microscopic fluctuations of the lamellipodia in the experiment.

The analytical solution of Eq. (5.3) for $\varrho(x, v, t)$ is not known, however, for large friction $\gamma_{\alpha}$ this equation boils down to a fractional diffusion equation for which $\varrho(x, t)$ can be calculated in terms of a Fox function [Sch89a]. The experimental data in Figs. 5.2 and 5.3 was then fitted consistently by using the above solutions with the four parameters $v_{t h}^{2}, \alpha, \gamma$ and $\eta^{2}$ in Bayesian data analysis [Die08].

In summary, by statistical analysis of experimental data we have shown that the equilibrium migration of the biological cells under consideration is anomalous. Related anomalies have also been observed for other types of migrating cells [Har94, Upa01, Li08, Tak08, Böd10]. Our experimental results are coherently reproduced by a mathematical model in form of a stochastic fractional equation. We now elaborate on possible physical and biological interpretations of our findings.

First of all, we remark that the solutions of Eq. (5.3) for both the MSD and the velocity autocorrelation function match precisely to the solutions of the generalized Langevin equation [Lut01]

$$
\dot{v}=-\int_{0}^{t} d t^{\prime} \gamma\left(t-t^{\prime}\right) v\left(t^{\prime}\right)+\xi(t) .
$$

Here $\xi(t)$ holds for Gaussian white noise and $\gamma(t) \sim t^{-\alpha}$ for a time-dependent friction coefficient with a power law memory kernel, which alternatively could be written by using a fractional derivative [Lut01]. For $\gamma(t) \sim \delta(t)$ the ordinary Langevin equation is recovered. Note that the position PDF generated by this equation is Gaussian in the long time limit 
and thus does not match to the one of the fractional Klein-Kramers equation Eq. (5.3). However, alternatively one could sample from a non-Gaussian $\xi(t)$ to generate a non-Gaussian position PDF. Strictly speaking, despite equivalent MSD and velocity correlations Eqs. (5.3) and (5.8) define different classes of anomalous stochastic processes. The precise cross-links between the Langevin description and the fractional Klein-Kramers equation are subtle [Eul07] and to some extent still unknown. The advantage of Eq. (5.8) is that it allows more straightforwardly a possible biophysical interpretation of the origin of the observed anomalous MSD and velocity correlations, at least partially, in terms of the existence of a memory-dependent friction coefficient. The latter, in turn, might be explained by anomalous rheological properties of the cell cytoskeleton, which consists of a complex biopolymer gel [Sem07].

Secondly, what could be the possible biological significance of the observed anomalous cell migration? There is an ongoing debate about whether biological organisms such as, e.g., albatrosses, marine predators and fruit flies have managed to mimimize the search time for food in a way that matches to optimizing search strategies in terms of stochastic processes; see Refs. [Bén11, Vis11] and further references therein. In particular, it has been argued that Lévy flights are superior to Brownian motion in order to find sparsely, randomly distributed, replenishing food sources [Bén11]. However, it was also shown that in other situations intermittent dynamics is more efficient than pure Lévy motion [Bén11]. For our cell experiment, both the experimental data and the theoretical modeling suggest that there exists a slow diffusion on short time scales, whereas the long-time motion is much faster, which resembles intermittency as discussed in Ref. [Bén11]. Hence, the results on anomalous cell migration presented above might be biologically relevant in view of suitably optimized foraging strategies.

\section{Summary}

This chapter highlighted some fundamental aspects of anomalous dynamics: The scene was set by section 2, which reviewed basic ideas of weak chaos by establishing crosslinks to infinite ergodic theory. This branch of ergodic theory provides a rigorous mathematical approach to study weakly chaotic dynamical systems. In particular, we proposed suitable definitions of generalized chaos quantities assessing weakly chaotic dynamics by yielding a generalized version of Pesin's theorem. We also outlined a generalized hierarchy of chaos on the basis of different functional forms of the dispersion exhibited by nearby trajectories of a deterministic dynamical system. In section 3 we related these concepts to the problem of anomalous diffusion by spatially extending our previously discussed simple map model. Applying stochastic continuous time random walk theory to this model in comparison to computer simulations, we learned about an intricate dynamical phase transition between normal and anomalous diffusion, governed by multiplicative logarithmic corrections in the mean square displacement. We also derived a fractional diffusion equation that reproduced the subdiffusive diffusive dynamics of this model on coarse scales. The subsequent section 4 elaborated on fluctuation relations, here understood as a large-deviation symmetry 
property of the work probability distributions generated by a given stochastic dynamics far from equilibrium. We familiarized ourselves with the conventional form of transient work fluctuation relations derived from standard Langevin dynamics before exploring anomalous generalizations of Langevin equations. One of them reproduced the conventional form of fluctuation relations, whereas the other one yielded a generalized, anomalous fluctuation relation. The precise form of the resulting fluctuation relation appeared to be intimately releated to whether or not fluctuation-dissipation relations are broken. In our final main section we related our previous theoretical ideas to the experimental problem of studying biological cell migration. By extracting the mean square displacement and the position probability distributions from experimental data, we found that the dynamics exhibited by these cells was anomalous, showing different behavior on different time scales, by eventually yielding superdiffusion for long times. On the basis of these experimental results we suggested a stochastic theoretical model of cell migration in form of a fractional Klein-Kramers equation, which coherently reproduced our experimental findings.

In summary, we traversed quite an anomalous scientific landscape of different but related topics: Starting from simple deterministic maps and their ergodic theory description we switched to basics of anomalous stochastic processes, studied both normal and anomalous stochastic fluctuations very far from equilibrium in terms of Langevin dynamics by ending up with anomalously crawling biological cells. We thus meant to illustrate the third column displayed in the very first Fig. 1.1 of the introduction, by also explaining the title of this contribution. Within a larger scientific context, one may consider our disucssion as an indication that a novel theory of anomalous nonequilibrium processes is presently emerging. In contrast to standard nonequilibrium statistical mechanics, this dynamics is inherently non-stationary, due to the weak chaos by which it is generated. This mechanism leads to important physical consequences like anomalous transport, which can be tested in experiments. On the side of theoretical physics this approach asks for further generalizations of recently developed fundamental concepts, perhaps leading to a weakly chaotic hypothesis, the identification of the physically relevant measures characterizing such systems, and to deriving experimentally measurable consequences such as generalizations of ordinary large deviation properties and fluctuation relations. However, these questions also motivate further mathematical work in upcoming directions of infinite ergodic theory to provide a formal framework and rigorous results for parts of the physical theory.

\section{Acknowledgements:}

Each of the four sections reflects the collaboration with colleagues, without whom the work presented here would not have been possible. The second section benefitted very much from discussion with R.Zweimüler, whom the author thanks very much for a lot of mathematical insight into aspects of infinite ergodic theory. Particularly, the author is indebted to his former postdoc P.Howard, who did brilliant work on calculating generalized chaos quantities for the Pomeau-Manneville map. Regarding the third section, credit goes to his former PhD student N.Korabel for joint work that formed part of his $\mathrm{PhD}$ thesis. A.V.Chechkin significantly contributed to the same section, as well as performing major research on the topic coverd by the fourth one. The author is deeply indebted to him for his long-term collaboration on anomalous stochastic processes. P.Dieterich was the driving force behind the project reviewed in the fifth section. The author thanks him for much insight into the biophysical aspects of biological cell migration. Finally, he wishes to thank the editors of this book for their patience with this book chapter. 


\section{Bibliography}

[Aar97] J. Aaronson, An introduction to infinite ergodic theory, volume 50 of Mathematical Surveys and Monographs. (American Mathematical Society, Providence, 1997).

[Aar00] J. Aaronson, An overview of infinite ergodic theory. in: M. Foreman et al., Eds., Descriptive set theory and dynamical systems, volume 277 of LMS lecture notes, pages 1-29, Cambridge University Press, Cambridge, 2000.

[Aki10] T. Akimotoa and Y. Aizawa, Subexponential instability in one-dimensional maps implies infinite invariant measure. Chaos 20, 033110/1-7 (2010).

[Ale11] A. Alemany, M. Ribezzi and F. Ritort, Recent progress in fluctuation theorems and free energy recovery. AIP Conference Proceedings 1332, 96-110 (2011).

[All97] K.T. Alligood, T.S. Sauer and J.A. Yorke, Chaos - An introduction to dynamical systems. (Springer, New York, 1997).

[Arn68] V.I. Arnold and A. Avez, Ergodic problems of classical mechanics. (W.A. Benjamin, New York, 1968).

[Art97] R. Artuso, G. Casati and I. Guarneri, Numerical study on ergodic properties of triangular billiards. Phys. Rev. E 55, 6384-6390 (1997).

[Art05] R. Artuso and G. Cristadoro, Anomalous deterministic transport. Chaos 15, 015116/1-7 (2005).

[Bad97] R. Badii and A. Politi, Complexity: hierarchical structures and scaling physics. (Cambridge University Press, Cambridge, 1997).

[Bal00] V. Baladi, Positive Transfer Operators and Decay of Correlations, volume 16 of Advanced Series in Nonlinear Dynamics. (World Scientific, Singapore, 2000).

[Bar00] E. Barkai and R. Silbey, Fractional Kramers Equation. J. Phys. Chem. B 104, 3866-3874 (2000).

[Bec93] C. Beck and F. Schlögl, Thermodynamics of Chaotic Systems, volume 4 of Cambridge nonlinear science series. (Cambridge University Press, Cambridge, 1993).

[Bec04] C. Beck and E.G.D. Cohen, Superstatistical generalization of the work fluctuation theorem. Physica A 344, 393-402 (2004).

[Bén11] O. Bénichou, C. Loverdo, M. Moreau and R. Voituriez, Intermittent search strategies. Rev. Mod. Phys. 83, 81 (2011). 
[Boc81a] G. Bochkov and Y. Kuzovlev, Nonlinear fluctuation-dissipation relations and stochastic models in nonequilibrium thermodynamics: I. Generalized fluctuationdissipation theorem. Physica A 106, 443-479 (1981).

[Boc81b] G. Bochkov and Y. Kuzovlev, Nonlinear fluctuation-dissipation relations and stochastic models in nonequilibrium thermodynamics: II. Kinetic potential and variational principles for nonlinear irreversible processes. Physica A 106, 480520 (1981).

[Böd10] H.U. Bödeker, C. Beta, T.D. Frank and E. Bodenschatz, Quantitative analysis of random ameboid motion. Europhys. Lett. 90, 28005/1-5 (2010).

[Bou90] J.-P. Bouchaud and A. Georges, Anomalous diffusion in disordered media: Statistical mechanisms, models and physical applications. Phys. Rep. 195, 127-293 (1990).

[Bus05] C. Bustamante, J. Liphardt and F. Ritort, The nonequilibrium thermodynamics of small systems. Phys. Today 58, 43-48 (2005).

[Cas08] C. Castiglione, M. Falcioni, Lesne A. and A. Vulpiani, Chaos and Coarse Graining in Statistical Mechanics. (Cambridge University Press, Cambridge, 2008).

[Cha08] S. Chaudhury, D. Chatterjee and B.J. Cherayil, Resolving a puzzle concerning fluctuation theorems for forced harmonic oscillators in non-Markovian heat baths. J. Stat. Mech.: Theor. Exp. 10, P10006/1-13 (2008).

[Che09] A.V. Chechkin and R. Klages, Fluctuation relations for anomalous dynamics. J. Stat. Mech.: Theor. Exp. 03, L03002/1-11 (2009).

[Cil10] S. Ciliberto, S. Joubaud and A. Petrosyan, Fluctuations in out-of-equilibrium systems: from theory to experiment. J. Stat. Mech. 2010, P12003/1-27 (2010).

[Cro99] G.E. Crooks, Entropy production fluctuation theorem and the nonequilibrium work relation for free energy differences. Phys. Rev. E 60, 2721-2726 (1999).

[Dev89] R.L. Devaney, An introduction to chaotic dynamical systems. (Addison-Wesley, Reading, second edition, 1989).

[Die08] P. Dieterich, R. Klages, R. Preuss and A. Schwab, Anomalous dynamics of cell migration. PNAS 105, 459-463 (2008).

[Dor99] J.R. Dorfman, An introduction to chaos in nonequilibrium statistical mechanics. (Cambridge University Press, Cambridge, 1999).

[Dun87] G.A. Dunn and A.F. Brown, A unified approach to analysing cell motility. J. Cell Sci. Suppl. 8, 81-102 (1987).

[Ebe05] W. Ebeling and I.M. Sokolov, Statistical thermodynamics and stochastic theory of nonequilibrium systems. (World Scientific, Singapore, 2005).

[Eck85] J.-P. Eckmann and D. Ruelle, Ergodic theory of chaos and strange attractors. Rev. Mod. Phys. 57, 617-656 (1985). 
[Esp08] M. Esposito and K. Lindenberg, Continuous-time random walk for open systems: Fluctuation theorems and counting statistics. Phys. Rev. E 77, 051119/1-12 (2008).

[Eul07] S. Eule, R. Friedrich, F. Jenko and D. Kleinhans, Langevin Approach to Fractional Diffusion Equations Including Inertial Effects. J. Phys. Chem. B 111, 1147411477 (2007).

[Eva93] D.J. Evans, E.G.D. Cohen and G.P. Morriss, Probability of second law violations in shearing steady flows. Phys. Rev. Lett. 71, 2401-2404 (1993).

[Eva94] D.J. Evans and D.J. Searles, Equilibrium microstates which generate second law violating steady states. Phys. Rev. E 50, 1645-1648 (1994).

[Eva02] D.J. Evans and D.J. Searles, The fluctuation theorem. Advances in Physics 51, 1529-1585 (2002).

[Eva05] D.J. Evans, D.J. Searles and L. Rondoni, Application of the Gallavotti-Cohen fluctuation relation to thermostated steady states near equilibrium. Phys. Rev. E 71, 056120/1-12 (2005).

[Fri10] P. Friedl and K. Wolf, Plasticity of cell migration: a multiscale tuning model. J. Cell Biol. 188, 11-19 (2010).

[Fuj82] H. Fujisaka and S. Grossmann, Diffusion in discrete nonlinear dynamical systems. Z. Physik B 48, 261-275 (1982).

[Gal95a] G. Gallavotti and E.G.D. Cohen, Dynamical ensembles in nonequilibrium statistical mechanics. Phys. Rev. Lett. 74, 2694-2697 (1995).

[Gal95b] G. Gallavotti and E.G.D. Cohen, Dynamical ensembles in stationary states. J. Stat. Phys. 80, 931-970 (1995).

[Gal98] G. Gallavotti, Chaotic dynamics, fluctuations, nonequilibrium ensembles. Chaos 8, 384-393 (1998).

[Gal03] S. Galatolo, Complexity, initial condition sensitivity, dimension and weak chaos in dynamical systems. Nonlinearity 16, 1219-1238 (2003).

[Gas88] P. Gaspard and X.-J. Wang, Sporadicity: Between periodic and chaotic dynamical behaviors. Proc. Nat. Acad. Sci. USA 85, 4591-4595 (1988).

[Gas98] P. Gaspard, Chaos, scattering, and statistical mechanics. (Cambridge University Press, Cambridge, 1998).

[Gei82] T. Geisel and J. Nierwetberg, Onset of diffusion and universal scaling in chaotic systems. Phys. Rev. Lett. 48, 7-10 (1982).

[Gei84] T. Geisel and S. Thomae, Anomalous diffusion in intermittent chaotic systems. Phys. Rev. Lett. 52, 1936-1939 (1984). 
[Gei85] T. Geisel, J. Nierwetberg and A. Zacherl, Accelerated diffusion in Josephson junctions and related chaotic systems. Phys. Rev. Lett. 54, 616-619 (1985).

[Gor97] R. Gorenflo and F. Mainardi, Differential Equations of Fractional Order. in: A. Carpinteri and F. Mainardi, Eds., Fractals and fractional calculus in continuum mechanics, volume 378 of CISM Courses and Lecture Notes, pages 223-276, Springer, Berlin, 1997.

[Har94] R.S. Hartmann, K. Lau, W. Chou and T.D. Coates, The fundamental motor of the human neutrophil is not random: Evidence for local non-Markov movement in neutrophils. Biophys. J. 67, 2535-2545 (1994).

[Har06] R.J. Harris, A. Rákos and G.M. Schütz, Breakdown of Gallavotti-Cohen symmetry for stochastic dynamics. Europhys. Lett. 75, 227-233 (2006).

[Har07] R.J. Harris and G.M. Schütz, Fluctuation theorems for stochastic dynamics. J. Stat. Mech. 7, P07020/1-45 (2007).

[Hat01] T. Hatano and S. Sasa, Steady-state thermodynamics of Langevin systems. Phys. Rev. Lett. 86, 3463-3466 (2001).

[How09] P.J. Howard and R. Klages, Entropy and stretching rates in intermittent maps. unpublished, 2009.

[Jar97a] C. Jarzynski, Equilibrium free-energy differences from nonequilibrium measurements: A master-equation approach. Phys. Rev. E 56, 5018-5035 (1997).

[Jar97b] C. Jarzynski, Nonequilibrium equality for free energy differences. Phys. Rev. Lett. 78, 2690-2693 (1997).

[Jar08] C. Jarzynski, Nonequilibrium work relations: foundations and applications. Eur. Phys. J. B 64, 331-340 (2008).

[Kat95] A. Katok and B. Hasselblatt, Introduction to the modern theory of dynamical systems, volume 54 of Encyclopedia of Mathematics and its Applications. (Cambridge University Press, Cambridge, 1995).

[Kel98] G. Keller, Equilibrium states in ergodic theory, volume 42 of London Mathematical Society Student Texts. (Cambridge University Press, Cambridge, 1998).

[Kla95] R. Klages and J.R. Dorfman, Simple maps with fractal diffusion coefficients. Phys. Rev. Lett. 74, 387-390 (1995).

[Kla96a] J. Klafter, M. F. Shlesinger and G. Zumofen, Beyond Brownian motion. Phys. Today 49, 33-39 (1996).

[Kla96b] R. Klages, Deterministic diffusion in one-dimensional chaotic dynamical systems. (Wissenschaft \& Technik-Verlag, Berlin, 1996).

[Kla97] R. Klages and J.R. Dorfman, Dynamical crossover in deterministic diffusion. Phys. Rev. E 55, R1247-R1250 (1997). 
[Kla99] R. Klages and J.R. Dorfman, Simple deterministic dynamical systems with fractal diffusion coefficients. Phys. Rev. E 59, 5361-5383 (1999).

[Kla07] R. Klages, Microscopic chaos, fractals and transport in nonequilibrium statistical mechanics, volume 24 of Advanced Series in Nonlinear Dynamics. (World Scientific, Singapore, 2007).

[Kla08] R. Klages, G. Radons and I.M. Sokolov, Eds., Anomalous Transport: Foundations and Applications. (Wiley-VCH, Berlin, 2008).

[Kla10] R. Klages, From Deterministic Chaos to Anomalous Diffusion. in: Reviews of Nonlinear Dynamics and Complexity, volume 3, pages 169-227, Wiley-VCH, Berlin, 2010.

[Kla11] R. Klages, R. Zweimüller, E. Barkai and H. Kantz, Weak Chaos, Infinite Ergodic Theory, and Anomalous Dynamics. http://www.pks.mpg.de/ wchaos11, 2011.

[Kla12a] R. Klages, A.V. Chechkin and P. Dieterich, Anomalous fluctuation relations. in: Nonequilibrium Statistical Physics of Small Systems: Fluctuation relations and beyond, Reviews of Nonlinear Dynamics and Complexity, pages 315-366, WileyVCH, Berlin, 2012. see Ref. [Kla12b].

[Kla12b] R. Klages, W. Just and C. Jarzynski, Eds., Nonequilibrium Statistical Physics of Small Systems: Fluctuation relations and beyond. Reviews of Nonlinear Dynamics and Complexity. (Wiley-VCH, Berlin, 2012). in print.

[Kor05] N. Korabel, A.V. Chechkin, R. Klages, I.M. Sokolov and V.Yu. Gonchar, Understanding anomalous transport in intermittent maps: From continuous time random walks to fractals. Europhys. Lett. 70, 63-69 (2005).

[Kor07] N. Korabel, R. Klages, A.V. Chechkin, I.M. Sokolov and V.Yu. Gonchar, Fractal properties of anomalous diffusion in intermittent maps. Phys. Rev. E 75, 036213/1-14 (2007).

[Kor09] N. Korabel and E. Barkai, Pesin-Type Identity for Intermittent Dynamics with a Zero Lyaponov Exponent. Phys. Rev. Lett. 102, 050601/1-4 (2009).

[Kor10] N. Korabel and E. Barkai, Separation of trajectories and its relation to entropy for intermittent systems with a zero Lyapunov exponent. Phys. Rev. E 82, 016209/1$11(2010)$.

[Kub66] R. Kubo, The Fluctuation-Dissipation Theorem. Rep. Prog. Phys. 29, 255-284 (1966).

[Kub92] R. Kubo, M. Toda and N. Hashitsume, Statistical Physics, volume 2 of Solid State Sciences. (Springer, Berlin, 2nd edition, 1992).

[Kur07] Jorge Kurchan, Non-equilibrium work relations. J. Stat. Mech.: Theor. Exp. 2007, P07005/1-14 (2007).

[Läm09] T. Lämmermann and M. Sixt, Mechanical modes of 'amoeboid' cell migration. Curr. Opin. Cell Biol. 21, 636-644 (2009). 
[Lau96] D.A. Lauffenburger and A.F.Horwitz, Cell migration: A physically integrated molecular process. Cell 84, 359-369 (1996).

[Li02] B. Li, L. Wang and B. Hu, Finite thermal conductivity in 1d models having zero Lyapunov exponent. Phys. Rev. Lett. 88, 223901/1-4 (2002).

[Li08] L. Li, S.F. Norrelykke and E.C. Cox, Persistent Cell Motion in the Absence of External Signals: A Search Strategy for Eukaryotic Cells. PLoS ONE 3, e2093/111 (2008).

[Lut01] E. Lutz, Fractional Langevin equation. Phys. Rev. E 64, 051106/1-4 (2001).

[Mai97] F. Mainardi, Fractional calculus, some basic problems in continuum and statistical mechanics. in: A. Carpinteri and F. Mainardi, Eds., Fractals and fractional calculus in continuum mechanics, volume 378 of CISM Courses and Lecture Notes, pages 291-348, Springer, Berlin, 1997.

[Mai07] T. Mai and A. Dhar, Nonequilibrium work fluctuations for oscillators in nonMarkovian baths. Phys. Rev. E 75, 061101/1-7 (2007).

[Mar02] D.S. Martin, M.B. Forstner and J.A. Käs, Apparent subdiffusion inherent to single particle tracking. Biophys. J. 83, 2109-2117 (2002).

[Met00] R. Metzler and J. Klafter, The random walk's guide to anomalous diffusion: $A$ fractional dynamics approach. Phys. Rep. 339, 1-77 (2000).

[Met04] R. Metzler and J. Klafter, The restaurant at the end of the random walk: Recent developments in the description of anomalous transport by fractional dynamics. J. Phys. A: Math. Gen. 37, R161-R208 (2004).

[Mon65] E.W. Montroll and G.H. Weiss, Random walks on lattices II. J. Math. Phys. 6, 167-179 (1965).

[Mon73] E.W. Montroll and H. Scher, Random walks on lattices IV: Continuous-time walks and influence of absorbing boundaries. J. Stat. Phys. 9, 101-133 (1973).

[Ohk07] T. Ohkuma and T. Ohta, Fluctuation theorems for non-linear generalized Langevin systems. J. Stat. Mech. 10, P10010/1-28 (2007).

[Ott93] E. Ott, Chaos in Dynamical Systems. (Cambridge University Press, Cambridge, 1993).

[Pan10] D. Panja, Anomalous polymer dynamics is non-Markovian: memory effects and the generalized Langevin equation formulation. J. Stat. Mech.: Theor. Exp. 06, P06011/1-34 (2010).

[Pod99] I. Podlubny, Fractional differential equations. (Academic Press, New York, 1999).

[Pom80] Y. Pomeau and P. Manneville, Intermittent transition to turbulence in dissipative dynamical systems. Commun. Math. Phys. 74, 189-197 (1980). 
[Por96] J.M. Porra, K.-G. Wang and J.Masoliver, Generalized Langevin equations: Anomalous diffusion and probability distributions. Phys. Rev. E 53, 5872-5881 (1996).

[Pre92] T. Prellberg and J. Slawny, Maps of intervals with indifferent fixed points: Thermodynamic formalism and phase transitions. J. Stat. Phys. 66, 503-514 (1992).

[Reb08] A. Rebenshtok and E. Barkai, Weakly Non-Ergodic Statistical Physics. J. Stat. Phys. 133, 565-586 (2008).

[Ris96] H. Risken, The Fokker-Planck Equation. (Springer, Berlin, 2nd edition, 1996).

[Rit03] F. Ritort, Work fluctuations, transient violations of the second law and free-energy recovery methods: Perspectives in theory and experiments. Poincaré Seminar 2, 195-229 (2003).

[Rob95] C. Robinson, Dynamical Systems. (CRC Press, London, 1995).

[Rue78] D. Ruelle, Thermodynamic Formalism, volume 5 of Encyclopedia of mathematics and its applications. (Addison-Wesley, Reading, Mass., 1978).

[Saa12] A. Saa and R. Venegeroles, Pesin-type relation for subexponential instability. J. Stat. Mech: Theor. Exp. 03, P03010/1-10 (2012).

[Sag10] T. Sagawa and M. Ueda, Generalized Jarzysnki equality under nonequilibrium feedback control. Phys. Rev. Lett. 104, 090602/1-4 (2010).

[Sch75] H. Scher and E.W. Montroll, Anomalous transit-time dispersion in amorphous solids. Phys. Rev. B 12, 2455-2477 (1975).

[Sch82] M. Schell, S. Fraser and R. Kapral, Diffusive dynamics in systems with translational symmetry: A one-dimensional-map model. Phys. Rev. A 26, 504-521 (1982).

[Sch89a] W.R. Schneider and W. Wyss, Fractional diffusion and wave equations. J. Math. Phys 30, 134-144 (1989).

[Sch89b] H.G. Schuster, Deterministic chaos. (VCH Verlagsgesellschaft mbH, Weinheim, 2nd edition, 1989).

[Sei05] U. Seifert, Entropy production along a stochastic trajectory and an integral fluctuation theorem. Phys. Rev. Lett. 95, 040602/1-4 (2005).

[Sei08] U. Seifert, Stochastic thermodynamics: principles and perspectives. Eur. Phys. J. B 64, 423-431 (2008).

[Sel98] M. Sellitto, Fluctuations of entropy production in driven glasses. preprint arXiv:qbio.PE/0404018, 1998.

[Sel09] M. Sellitto, Fluctuation relation and heterogeneous superdiffusion in glassy transport. Phys. Rev. E 80, 011134/1-5 (2009). 
[Sem07] C. Semmrich, T. Storz, J. Glaser, R. Merkel, A.R. Bausch and K. Kroy, Glass transition and rheological redundancy in F-actin solutions. PNAS 104, 2019920203 (2007).

[Shl85] M.F. Shlesinger and J. Klafter, Accelerated diffusion in Josephson-junctions and related chaotic systems - comment. Phys. Rev. Lett. 54, 2551 (1985).

[Shl93] M.F. Shlesinger, G.M. Zaslavsky and J. Klafter, Strange kinetics. Nature 363, 31-37 (1993).

[Sok02] I.M. Sokolov, J. Klafter and A. Blumen, Fractional kinetics. Phys. Today 55, 48-54 (2002).

[Ste09] F.D. Stefani, J.P. Hoogenboom and E. Barkai, Beyond quantum jumps: Blinking nanoscale light emitters. Phys. Today 62, 34-39 (2009).

[Sto91] C.L. Stokes and S.K. Williams D.A. Lauffenburger, Migration of individual microvessel endothelial cells: Stochastic model and parameter measurement. J. Cell Science 99, 419-430 (1991).

[Tak08] H. Takagi, M.J. Sato, T. Yanagida and M. Ueda, Functional Analysis of Spontaneous Cell Movement under Different Physiological Conditions. PLoS ONE 3, e2648/1-8 (2008).

[Tal10] A. Taloni, A.V. Chechkin and J. Klafter, Generalized Elastic Model Yields a Fractional Langevin Equation Description. Phys. Rev. Lett. 104, 160602/1-4 (2010).

[Tas02] S. Tasaki and P. Gaspard, Spectral properties of a piecewise linear intermittent map. J. Stat. Phys. 109, 803-820 (2002).

[Tas04] S. Tasaki and P. Gaspard, Spectral characterization of anomalous diffusion of a periodic piecewise linear intermittent map. Physica D 187, 51-65 (2004).

[Tha83] M. Thaler, Transformations on [0,1] with infinite invariant measures. Israel J. Math. 46, 67-96 (1983).

[Tha01] M. Thaler, Infinite ergodic theory. course notes from "The Dynamic Odyssey", CIRM 2001, available at http://www.sbg.ac.at/mat/staff/thaler/thaler_english.htm, 2001.

[Tha06] M. Thaler and R. Zweimüller, Distributional limit theorems in infinite ergodic theory. Probab. Theory Relat. Fields 155, 15-52 (2006).

[Tod92] M. Toda, R. Kubo and N. Saitô, Statistical Physics, volume 1 of Solid State Sciences. (Springer, Berlin, 2nd edition, 1992).

[Tou07] H. Touchette and E.G.D. Cohen, Fluctuation relation for a Lévy particle. Phys. Rev. E 76, 020101(R)/1-4 (2007).

[Tou09] H. Touchette and E.G.D. Cohen, Anomalous fluctuation properties. Phys. Rev. E 80, 011114/1-11 (2009). 
[Toy10] Shoichi Toyabe, Takahiro Sagawa, Masahito Ueda, Eiro Muneyuki and Masaki Sano, Experimental demonstration of information-to-energy conversion and validation of the generalized Jarzynski equality. Nature Physics 6, 988-992 (2010).

[Upa01] A. Upadhyaya, J.P. Rieu, J.A. Glazier and Y. Sawada, Anomalous diffusion and non-Gaussian velocity distributions of Hydra cells in cellular aggregates. Physica A 293, 549-558 (2001).

[vB04] H. van Beijeren, Generalized dynamical entropies in weakly chaotic systems. Physica D 193, 90-95 (2004).

[Vis11] G.M Viswanathan, M.G.E. da Luz, E.P. Raposo and H.E. Stanley, The Physics of Foraging. (Cambridge University Press, Cambridge, 2011).

[vZ03a] R. van Zon and E.G.D. Cohen, Extension of the fluctuation theorem. Phys. Rev. Lett. 91, 110601/1-4 (2003).

[vZ03b] R. van Zon and E.G.D. Cohen, Stationary and transient work-fluctuation theorems for a dragged Brownian particle. Phys. Rev. E 67, 046102/1-10 (2003).

[Wan89] X.J. Wang, Abnormal fluctuations and thermodynamic phase transitions In dynamical systems. Phys. Rev. A 39, 3214-3217 (1989).

[Wan02] G.M. Wang, E.M. Sevick, E. Mittag, D.J. Searles and D.J. Evans, Experimental demonstration of violations of the second law of thermodynamics for small systems and short time scales. Phys. Rev. Lett. 89, 050601/1-4 (2002).

[Wei94] G.H. Weiss, Aspects and applications of the random walk. (North-Holland, Amsterdam, 1994).

[You02] L.-S. Young, What are SRB measures, and which dynamical systems have them? J. Stat. Phys. 108, 733-754 (2002).

[Zam05a] F. Zamponi, F. Bonetto, L.F. Cugliandolo and J. Kurchan, A fluctuation theorem for non-equilibrium relaxational systems driven by external forces. J. Stat. Mech.: Theor. Exp. 09, P09013/1-51 (2005).

[Zam05b] F. Zamponi, G. Ruocco and L. Angelani, Generalized fluctuation relation and effective temperature in a driven fluid. Phys. Rev. E 71, 020101(R)/1-4 (2005).

[Zas01] G.M. Zaslavsky and D.A. Usikov, Weak chaos and quasi-regular patterns. Cambridge Nonlinear Science Series. (Cambridge University Press, Cambridge, 2001).

[Zas02] G.M. Zaslavsky, Chaos, fractional kinetics, and anomalous transport. Phys. Rep. 371, 461-580 (2002).

[Zas03] G.M. Zaslavsky and M. Edelman, Pseudochaos. in: E. Kaplan and J.E. Marsden, Eds., Perspective and problems in nonlinear science, pages 421-443, Springer, New York, 2003. 
[Zas05] G.M. Zaslavsky, B.A. Carreras, V.E. Lynch, L. Garcia and M. Edelman, Topological instability along invariant surfaces and pseudochaotic transport. Phys. Rev. E 72, 026227/1-11 (2005).

[Zum93] G. Zumofen and J. Klafter, Scale-invariant motion in intermittent chaotic systems. Phys. Rev. E 47, 851-863 (1993).

[Zwe98] R. Zweimüller, Ergodic structure and invariant densities of non-Markovian interval maps with indifferent fixed points. Nonlinearity 11, 1263-1276 (1998).

[Zwe00] R. Zweimüller, Ergodic properties of infinite measure-preserving interval maps with indifferent fixed points. Ergod. Theory Dyn. Syst. 20, 1519-1549 (2000).

[Zwe09] R. Zweimüller, Surrey notes on infinite ergodic theory. course notes from the "LMS Graduate school on Ergodic Theory", Surrey, 2009, available at http://homepage.univie.ac.at/roland.zweimueller/MyPub/SurreyNotes.pdf, 2009 . 